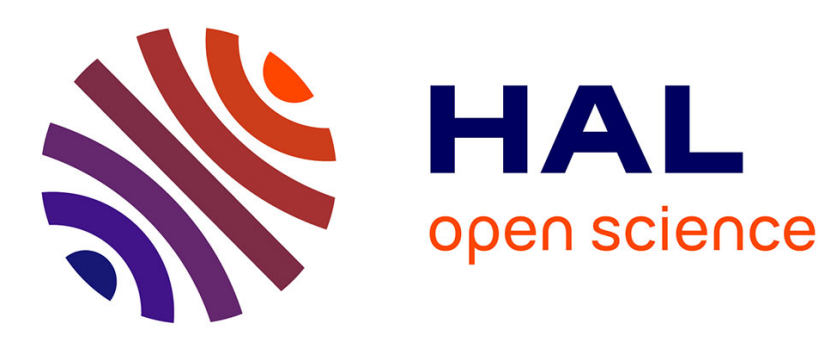

\title{
Maximum likelihood estimation of stochastic chaos representations from experimental data
}

\author{
Christophe Desceliers, R. Ghanem, Christian Soize
}

\section{To cite this version:}

Christophe Desceliers, R. Ghanem, Christian Soize. Maximum likelihood estimation of stochastic chaos representations from experimental data. International Journal for Numerical Methods in Engineering, 2006, 66 (6), pp.978-1001. 10.1002/nme.1576 . hal-00686154

\section{HAL Id: hal-00686154 \\ https://hal.science/hal-00686154}

Submitted on 7 Apr 2012

HAL is a multi-disciplinary open access archive for the deposit and dissemination of scientific research documents, whether they are published or not. The documents may come from teaching and research institutions in France or abroad, or from public or private research centers.
L'archive ouverte pluridisciplinaire HAL, est destinée au dépôt et à la diffusion de documents scientifiques de niveau recherche, publiés ou non, émanant des établissements d'enseignement et de recherche français ou étrangers, des laboratoires publics ou privés. 


\title{
Maximum Likelihood Estimation of Stochastic Chaos Representations from Experimental Data
}

\author{
Christophe Desceliers ${ }^{1}$, Roger Ghanem ${ }^{2},{ }^{*}$ and Christian Soize ${ }^{1}$ \\ ${ }^{1}$ Université Marne-La-Vallée, Paris, France \\ 210 KAP Hall, University of Southern California, Los Angeles, CA 90089.
}

KEY WORDS: Stochastic systems; Stochastic Estimation; Stochastic Inverse Analysis; Estimation of Chaos Coefficients

\section{SUMMARY}

This paper deals with the identification of probabilistic models of the random coefficients in stochastic boundary value problems (SBVP). The data used in the identification correspond to measurements of the displacement field along the boundary of domains subjected to specified external forcing. Starting with a particular mathematical model for the mechanical behavior of the specimen, the unknown field to be identified is projected on an adapted functional basis such as a provided by a finite element discretization. For each set of measurements of the displacement field along the boundary, an inverse problem is formulated to calculate the corresponding optimal realization of the coefficients of the unknown random field on the adapted basis. Realizations of these coefficients are then used, in conjunction with the maximum likelihood principle, to set-up and solve an optimization problem for the estimation of the coefficients in a polynomial chaos representation of the parameters of the SBVP. Copyright (c) 2004 John Wiley \& Sons, Ltd.

\section{Introduction}

In many problems of science and engineering, the physical systems under consideration can be modeled by boundary value problems whose parameters are random variables or stochastic fields. A fundamental difficulty in connection with these problems is the experimental identification of probabilistic models of these random parameters. Although stochastic inverse problems, in general, have received considerable attention over the past decade by engineers, scientists and mathematicians $[1,2,3,4,5]$, the particular class of problems addressed in this paper, and pertaining to the experimental identification of stochastic process models of parameters in random boundary value problems, has remained largely unexplored.

This paper deals with the identification of a probabilistic model of the random coefficients of stochastic differential operators for boundary value problems. A concrete example would be the experimental identification of the random field modeling the Young modulus of a

\footnotetext{
${ }^{*}$ Correspondence to: Roger Ghanem, 210 KAP Hall, University of Southern California, Los Angeles, CA 90089.
} 
random linear isotropic elastic heterogeneous medium. The data on which the identification is based correspond to measurements of the displacement field along the boundary of specimens submitted to a given external load. Briefly, the proposed method consists of first estimating the optimal spatial distribution of the model parameters, one realization at a time. This is accomplished through an inverse analysis which involves projecting the unknown field to be identified (for instance the Young modulus) on an adapted functional basis (for instance the finite element basis). This series of inverse problems results in a statistical sample consisting of realizations of the random parameter. These parameters are then represented by using the chaos decomposition $[6,7,8]$ and invoking a second optimization problem involving the maximum likelihood principle. The end product is a set of deterministic coefficients which provides a chaos representation of the random model parameters that is statistically consistent with the mechanical model and the experimental measurements.

A numerical application is presented in order to exemplify the proposed procedure. In particular a three-dimensional elastic linear isotropic heterogeneous elastic bounded domain subjected to static loads is considered. The sole random parameter of this random medium is the Young modulus which is modeled as a random field. A chaos representation of this random field is constructed from an experimental database previously constructed via numerical simulation and consisting of the displacement field on the boundary of the elastic random medium. A convergence analysis is also performed.

\section{Representation of the Stochastic Field}

Consider the second-order $\mathbb{R}^{n}$-valued stochastic field

$$
\boldsymbol{U}(\boldsymbol{x})=\left(U_{1}(\boldsymbol{x}), \ldots, U_{n}(\boldsymbol{x})\right) \quad x \in \Xi \subset \mathbb{R}^{d}
$$

indexed by some set $\Xi$ in $\mathbb{R}^{d}$, with zero mean and which is mean-square continuous on $\Xi$. We are interested in constructing representations of the stochastic field $\boldsymbol{U}(\boldsymbol{x})$ on a bounded and closed subset $\mathcal{D}$ in $\Xi$. This construction permits the treatment of the case where $\boldsymbol{U}(\boldsymbol{x})$ is stationary, associating $\Xi$ to the whole of $\mathbb{R}^{d}$. A first step is to develop a representation of random field $\boldsymbol{U}(\boldsymbol{x})$ in terms of a denumerable set of random variables. These random variables can be viewed as the projections of $\boldsymbol{U}$ on a hilbertian basis $\left\{\boldsymbol{v}_{i}\right\}$. These projections are secondorder random variables and are in general correlated and represent, in some sense, the original stochastic field.

In the next subsection, the representation of stochastic field $\boldsymbol{U}$ is developed for a general hilbertian basis. The following subsection demonstrates the development for the important special cases where these bases are obtained from the eigenvalue problem associated with any self-adjoint operator or from the eigenvalue problem associated with the covariance operator of random field $\boldsymbol{U}$. The representation of stochastic field $\boldsymbol{U}$ associated with this latter basis is also known as the Karhunen-Loeve expansion. Properties of the general representations are derived at the end of the section.

\subsection{Representation on Hilbertian Bases}

In general, stochastic field $\boldsymbol{U}$ of interest is not directly observable. Rather, it is indirectly obtained by solving an inverse problem that represents the measuring process. Examples of 
this procedure include estimating elasticity constants, $\boldsymbol{U}(\boldsymbol{x})$, from displacement measurements on the boundary of a nonhomogeneous specimen, or estimating the strain field, $\boldsymbol{U}(\boldsymbol{x})$, from its locally averaged measurements. The structure of the information associated with this type of inverse problem justifies the estimation of generalized representators of $\boldsymbol{U}(\boldsymbol{x})$ from which the physical pointwise representation can be subsequently synthesized. These generalized coordinates can be constructed as projections in a suitable Hilbert space $H$.

Let $H$ be a Hilbert space of functions $\boldsymbol{x} \mapsto \boldsymbol{v}(\boldsymbol{x})$ defined on $\mathcal{D}$ with values in $\mathbb{R}^{n}$, and denote the inner product on $H$ by $\langle\boldsymbol{v}, \boldsymbol{w}\rangle_{H}$, Let $\left\{\boldsymbol{v}_{j}, j=1, \ldots,+\infty\right\}$ be a hilbertian basis in $H$, which is thus a complete orthonormal system in $H$,

$$
\left\langle\boldsymbol{v}_{j}, \boldsymbol{v}_{k}\right\rangle_{H}=\delta_{j k}
$$

where $\delta_{j k}$ denotes the kronecker delta function. Stochastic field $\boldsymbol{U}(\boldsymbol{x})$ can then be written as,

$$
\boldsymbol{U}(\boldsymbol{x})=\sum_{j=1}^{\infty} \sqrt{\lambda_{j}} \eta_{j} \boldsymbol{v}_{j}(\boldsymbol{x})
$$

where, $\eta_{1}, \eta_{2} \ldots$, are centered second-order random variables, generally correlated, such that

$$
E\left\{\eta_{j}^{2}\right\}=1, \quad \forall j \geq 1 .
$$

and where

$$
\lambda_{1} \geq \lambda_{2} \geq \ldots \rightarrow 0
$$

is a decreasing sequence of positive real numbers such that

$$
\sum_{j=1}^{\infty} \lambda_{j}<+\infty,
$$

The series on the right-hand side of Equation (??) converges in mean-square sense in $H$. In this case, the following equality holds,

$$
E\left\{\|\boldsymbol{U}\|_{H}^{2}\right\}=\sum_{j=1}^{\infty} \lambda_{j},
$$

where $E\{\}$ denotes the operator of mathematical expectation. An expression for $\eta_{j}$ can then be written in the form,

$$
\eta_{j}=\frac{1}{\sqrt{\lambda_{j}}}\left\langle\boldsymbol{U}, \boldsymbol{v}_{j}\right\rangle_{H}
$$

\subsection{Special Cases}

Two particular cases of hilbertian bases can be associated with common experimental procedures. The first one corresponds to the situation where the covariance function of stochastic field $\{\boldsymbol{U}(\boldsymbol{x}), \quad \boldsymbol{x} \in \mathcal{D}\}$ can be accurately estimated either from direct measurements or indirect measurements following an inverse problem. In this case the eigenvectors of the covariance operator can be used as hilbertian basis $\left\{\boldsymbol{v}_{j}\right\}$. In the second case, the covariance function cannot be estimated in the physical coordinate system. In this case, an associated eigenvalue problem, adapted to the physical problem at hand, has to be introduced for constructing hilbertian basis $\left\{\boldsymbol{v}_{j}\right\}$. These two cases are addressed, respectively, in the following two sections. 
2.2.1. Karhunen-Loeve Expansion. The Karhunen-Loeve development for this $\{\boldsymbol{U}(\boldsymbol{x}), x \in \mathcal{D}\}$ provides a representation of $\boldsymbol{U}$ with a number of interesting properties. It is in fact optimal in the mean-square sense and yields a sequence of random variables that, while uncorrelated, are generally statistically dependent.

Working in Hilbert space $H=L^{2}\left(\mathcal{D}, \mathbb{R}^{n}\right)$ with the inner product given by

$$
\langle\boldsymbol{v}, \boldsymbol{w}\rangle_{H}=\int_{\mathcal{D}}\langle\boldsymbol{v}(\boldsymbol{x}), \boldsymbol{w}(\boldsymbol{x})\rangle_{\mathbb{R}^{n}} d \boldsymbol{x},
$$

the Karhunen-Loeve expansion can then be obtained by solving the following eigenvalue problem,

$$
\boldsymbol{R}_{\boldsymbol{U}} \boldsymbol{v}=\lambda \boldsymbol{v}
$$

where $\boldsymbol{R}_{\boldsymbol{U}}$ is the covariance operator in $H$ defined by the positive symmetric bilinear form on $H \times H$

$$
\left\langle\boldsymbol{R}_{\boldsymbol{U}} \boldsymbol{v}, \boldsymbol{w}\right\rangle_{H}=\int_{\mathcal{D}} \int_{\mathcal{D}}\left\langle\left[R_{\boldsymbol{U}}\left(\boldsymbol{x}, \boldsymbol{x}^{\prime}\right)\right] \boldsymbol{v}\left(\boldsymbol{x}^{\prime}\right), \boldsymbol{w}(\boldsymbol{x})\right\rangle_{\mathbb{R}^{n}} d \boldsymbol{x} d \boldsymbol{x}^{\prime}
$$

in which $\left[R_{\boldsymbol{U}}\left(\boldsymbol{x}, \boldsymbol{x}^{\prime}\right)\right]$ is the matrix of covariances of $\boldsymbol{U}(\boldsymbol{x})$,

$$
\left[R_{\boldsymbol{U}}\left(\boldsymbol{x}, \boldsymbol{x}^{\prime}\right)\right]=E\left\{\boldsymbol{U}(\boldsymbol{x}) \boldsymbol{U}\left(\boldsymbol{x}^{\prime}\right)^{T}\right\} .
$$

Since $\boldsymbol{U}(\boldsymbol{x})$ is assumed to be mean-square continuous on $\Xi$, the covariance function $\left[R_{\boldsymbol{U}}\left(\boldsymbol{x}, \boldsymbol{x}^{\prime}\right)\right]$ is continuous on $\Xi \times \Xi$ and consequently, it is square-integrable on $\mathcal{D} \times \mathcal{D}$ since $\mathcal{D}$ is a compact set. Thus, $\boldsymbol{R}_{\boldsymbol{U}}$ as defined above is Hilbert-Schmidt, and therefore its eigenvalues form a decreasing sequence of positive numbers, $\lambda_{1} \geq \ldots \geq \lambda_{n} \geq \ldots \rightarrow 0$, and its eigenvectors form a complete orthonormal system in $H$,

$$
\left\langle\boldsymbol{v}_{j}, \boldsymbol{v}_{k}\right\rangle_{H}=\delta_{j k} .
$$

Moreover, for every $\boldsymbol{x}$ and $\boldsymbol{x}^{\prime}$ in $\mathcal{D}$,

$$
\left[R_{\boldsymbol{U}}\left(\boldsymbol{x}, \boldsymbol{x}^{\prime}\right)\right]=\sum_{j=1}^{\infty} \lambda_{j} \boldsymbol{v}_{j}(\boldsymbol{x}) \boldsymbol{v}_{j}\left(\boldsymbol{x}^{\prime}\right)^{T}
$$

in which the series on the right-hand side is uniformly convergent on $\mathcal{D} \times \mathcal{D}$. Furthermore, it can be shown that

$$
E\left\{\|\boldsymbol{U}\|_{H}^{2}\right\}=\int_{\mathcal{D}} \operatorname{tr}\left[R_{\boldsymbol{U}}(\boldsymbol{x}, \boldsymbol{x})\right] d \boldsymbol{x}=\sum_{j=1}^{\infty} \lambda_{j}<+\infty .
$$

It is noted that since the left-hand side of Equation (??) is finite, the series on the righthand side is also finite and the operator $\boldsymbol{R}_{U}$ is nuclear. The resulting expansion given by Equation (??) is referred to as the Karhunen-Loeve expansion. As noted above, the series in this representation converges in mean-square sense in $H$. It is also noted that since the covariance function of $\boldsymbol{U}$ is continuous, this convergence is uniform at every $\boldsymbol{x} \in \mathcal{D}$. In addition, the random variables $\left\{\eta_{j}\right\}$, given by Equation (??), are second-order centered orthogonal random variables,

$$
E\left\{\eta_{j}\right\}=0 \quad \forall j \quad E\left\{\eta_{j} \eta_{k}\right\}=\delta_{j k} .
$$

It is again emphasized that these random variables are not, in general, statistically independent. 
2.2.2. Hilbertian Basis from a Self-Adjoint Operator. A generalization of the previous treatment is possible by considering representation on more general basis functions constructed as the eigenfunctions of a self-adjoint operator, $\boldsymbol{A}$. This operator, defined in $H$, is generally unbounded and admits a countable sequence of real eigenvalues $s$. This eigenvalue problem could, for example, be associated with an underlying boundary value problem that involves random field $\boldsymbol{U}$ as a parameter. This boundary value problem can be either derived from the physical problem itself or from a reduced model that is more efficient at representing the measurement process.

Thus a hilbertian basis is obtained as the solution $\left\{\boldsymbol{v}_{j}\right\}$ of the eigenvalue problem,

$$
\boldsymbol{A} \boldsymbol{v}=s \boldsymbol{v} .
$$

At this point, the development in the previous subsection for representing random field $\boldsymbol{U}$ in the coordinate system $\left\{\boldsymbol{v}_{j}\right\}$ can be readily implemented. In particular, the scalar $\lambda_{j}$ introduced in Section (??) is the square of the magnitude of the projection of $\boldsymbol{U}$ on coordinate $\boldsymbol{v}_{j}$ as indicated by Equation (??). In general, however, and except for the Karhunen-Loeve representation, convergence of representations with respect to hilbertian bases is not uniform, and the associated random variables are not orthogonal.

\subsection{Finite-Dimensional Approximation and Error Estimation}

A truncated expansion on hilbertian bases is commonly used in computationally targeted representations of stochastic fields. Truncating the expansion of $\boldsymbol{U}(\boldsymbol{x})$ at the $\mu^{\text {th }}$ term results in the following approximation to the field,

$$
\boldsymbol{U}^{\mu}(\boldsymbol{x})=\sum_{j=1}^{\mu} \sqrt{\lambda_{j}} \eta_{j} \boldsymbol{v}_{j}(\boldsymbol{x})
$$

incurring a random error $\epsilon_{\mu}(\boldsymbol{x})$ given by

$$
\epsilon_{\mu}(\boldsymbol{x})=\sum_{j=\mu+1}^{\infty} \sqrt{\lambda_{j}} \eta_{j} \boldsymbol{v}_{j}(\boldsymbol{x})
$$

which converges in mean-square sense in $H$ to zero as $\mu \rightarrow \infty$. Note that for the KarhunenLoeve case, this convergence is at every $\boldsymbol{x} \in \mathcal{D}$. Moreover it is noted that,

$$
E\left\{\left\|\boldsymbol{U}^{\mu}\right\|_{H}^{2}\right\}=\sum_{j=1}^{\mu} \lambda_{j}
$$

and thus,

$$
E\left\{\left\|\boldsymbol{U}-\boldsymbol{U}^{\mu}\right\|_{H}^{2}\right\}=\sum_{j=\mu+1}^{\infty} \lambda_{j}
$$

with the above series converging to zero as $\mu \rightarrow \infty$. Using Equation (??), the above norm of the error can be expressed as,

$$
E\left\{\left\|\boldsymbol{U}-\boldsymbol{U}^{\mu}\right\|_{H}^{2}\right\}=\int_{\mathcal{D}} \operatorname{tr}\left[R_{\boldsymbol{U}}(\boldsymbol{x}, \boldsymbol{x})\right] d \boldsymbol{x}-\sum_{j=1}^{\mu} \lambda_{j} .
$$

Equation (??) permits the estimation of the norm of the error associated with a given level of truncation, from a knowledge of the covariance function of $\boldsymbol{U}(\boldsymbol{x})$ and $\mu$ basis functions, $\boldsymbol{v}_{1}, \ldots, \boldsymbol{v}_{\mu}$. 


\subsection{Probability Distribution of a Finite Approximating Sequence}

For all $\boldsymbol{b}$ in $\mathbb{R}^{\mu}$, the characteristic functional of the $\mathbb{R}^{\mu}$-valued random variables $\boldsymbol{\eta}=\left(\eta_{1}, \ldots, \eta_{\mu}\right)$ appearing in Equation (??) can be written as,

$$
\begin{aligned}
\Psi_{\boldsymbol{\eta}}(\boldsymbol{b}) & =E\left\{e^{i\langle\boldsymbol{\eta}, \boldsymbol{b}\rangle_{\mathbb{R}} \mu}\right\} \\
& =E\left\{e^{i\left\langle\boldsymbol{U}, \sum_{j=1}^{\mu} \frac{1}{\sqrt{\lambda_{j}}} \boldsymbol{v}_{j} b_{j}\right\rangle_{H}}\right\} \\
& =E\left\{e^{i\langle\boldsymbol{U}, \boldsymbol{\zeta}\rangle_{H}}\right\}
\end{aligned}
$$

for $\zeta$ in a finite subspace of $H$ written as,

$$
\boldsymbol{\zeta}(\boldsymbol{x})=\sum_{j=1}^{\mu} \frac{1}{\sqrt{\lambda_{j}}} b_{j} \boldsymbol{v}_{j}(\boldsymbol{x}) .
$$

The following relationship is thus deduced between the characteristic functional $\Psi_{\boldsymbol{\eta}}(\boldsymbol{b})$ of $\boldsymbol{\eta}$ and the characteristic functional $\Psi_{\boldsymbol{U}}(\boldsymbol{\zeta})$ of $\boldsymbol{U}$,

$$
\begin{aligned}
\Psi_{\boldsymbol{\eta}}(\boldsymbol{b}) & =\Psi_{\boldsymbol{U}}(\boldsymbol{\zeta}) \\
& =E\left\{e^{i\langle\boldsymbol{U}, \boldsymbol{\zeta}\rangle_{H}}\right\} .
\end{aligned}
$$

It is noted that the characteristic functional $\Psi_{\boldsymbol{U}}(\boldsymbol{\zeta})$ of $\boldsymbol{U}$, defined on an appropriate space, completely defines the probability law of stochastic field $\boldsymbol{U}$. From the above development, this functional also completely characterizes the characteristic functional of the finite sequence of random variables $\boldsymbol{\eta}=\left(\eta_{1}, \ldots, \eta_{\mu}\right)$ for any value of $\mu<\infty$.

It is recalled that if $\Psi_{\eta}$ is an integrable function on $\mathbb{R}^{\mu}$, then random variable $\boldsymbol{\eta}$ is defined by a probability density function $p_{\boldsymbol{\eta}}(\boldsymbol{y})$ with respect to $d \boldsymbol{y}$ which is given by,

$$
p_{\boldsymbol{\eta}}(\boldsymbol{y})=\frac{1}{(2 \pi)^{\mu}} \int_{\mathbb{R}^{\mu}} e^{-i\langle\boldsymbol{y}, \boldsymbol{b}\rangle_{\mathbb{R}}^{\mu}} \Psi_{\boldsymbol{\eta}}(\boldsymbol{b}) d \boldsymbol{b} .
$$

It is also noted from Equation (??) that if $\boldsymbol{U}$ is a Gaussian stochastic field, then $\boldsymbol{\eta}$ is a Gaussian random vector.

\subsection{Example of a finite-dimensional construction using a finite element basis}

In general, a finite-dimensional approximation of the decomposition defined by Eq. (??) has to be constructed. Typically, domain $\mathcal{D}$ is a bounded domain discretized by the finite element method. Therefore, a finite element representation of random field $\{\boldsymbol{U}(\boldsymbol{x}), \boldsymbol{x} \in \mathcal{D}\}$ is constructed such that

$$
\boldsymbol{U}(\boldsymbol{x}) \simeq \sum_{k=1}^{N_{i}} \widetilde{\boldsymbol{U}}_{k} h_{k}(\boldsymbol{x}),
$$

in which $h_{1}(\boldsymbol{x}), \ldots, h_{N_{i}}(\boldsymbol{x})$ are the functions which are constructed from the interpolation functions of the finite elements used, where $N_{i}$ is an integer related to the degree of this 
approximation and to the mesh size and where $\left\{\widetilde{\boldsymbol{U}}_{1}, \ldots, \widetilde{\boldsymbol{U}}_{N_{i}}\right\}$ are the values of $\boldsymbol{U}(\boldsymbol{x})$ at the different nodes of the finite element mesh of domain $\mathcal{D}$. Let $\widetilde{\boldsymbol{\eta}}=\left(\widetilde{\boldsymbol{U}}_{1}, \ldots, \widetilde{\boldsymbol{U}}_{N_{i}}\right)$ be the centered second-order $\mathbb{R}^{n N_{i}}$-valued random variable whose covariance matrix is defined by $\left[C_{\tilde{\boldsymbol{\eta}}}\right]=E\left\{\widetilde{\boldsymbol{\eta}} \widetilde{\boldsymbol{\eta}}^{T}\right\}$. It should be noted that $\boldsymbol{U}(\boldsymbol{x})$ being a random vector with values in $\mathbb{R}^{n}$, $\widetilde{\boldsymbol{U}}_{1}, \ldots, \widetilde{\boldsymbol{U}}_{N_{i}}$ are also random vectors with values in $\mathbb{R}^{n}$ and consequently, $\widetilde{\boldsymbol{\eta}}$ is a random vector with values in $\mathbb{R}^{n N_{i}}$. Let us consider the eigenvalue problem $\left[C_{\tilde{\boldsymbol{\eta}}}\right] \boldsymbol{F}_{j}=\lambda_{j} \boldsymbol{F}_{j}$ for positive symmetric matrix $\left[C_{\tilde{\eta}}\right]$ for which the positive eigenvalues $\lambda_{1} \geq \lambda_{2} \geq \ldots \geq \lambda_{\mu} \geq \ldots$ are ordered in descending order. The associated eigenvectors $\boldsymbol{F}_{1}, \boldsymbol{F}_{2}, \ldots$, are normalized to one. Therefore, it can be written that $\widetilde{\boldsymbol{\eta}} \simeq \widetilde{\boldsymbol{\eta}}^{\mu}=\sum_{j=1}^{\mu} \sqrt{\lambda_{j}} \eta_{j} \boldsymbol{F}_{j}$ in which the real-valued random variables $\left\{\eta_{1}, \ldots, \eta_{\mu}\right\}$ defined by $\sqrt{\lambda_{j}} \eta_{j}=\left\langle\widetilde{\boldsymbol{\eta}}, \boldsymbol{F}_{j}\right\rangle_{\mathbb{R}^{n N_{i}}}$ are such that, for all $j$ and $k, E\left\{\eta_{j}\right\}=0$ and $E\left\{\eta_{j} \eta_{k}\right\}=\delta_{j k}$. The finite-dimensional representation associated with Eq. (??) can then be deduced.

\section{Properties of the Chaos Decomposition of the Finite Dimensional Approximation}

In this section the Chaos representation of the random variable $\boldsymbol{\eta}$ is used to derive the Chaos decomposition of random field $\boldsymbol{U}^{\mu}$. A-posteriori error estimators corresponding to a finite dimensional Chaos representation are also derived along with representations of the associated characteristic functionals.

\subsection{Chaos Representation}

In general, as explained in Section (??), second-order $\mathbb{R}^{\mu}$-valued random vector $\boldsymbol{\eta}$, while orthonormal, has an arbitrary probability distribution depending on the probability law of random process $\boldsymbol{U}$. It is assumed, however, that there exists a mapping $\boldsymbol{g}: \mathbb{R}^{\nu} \longrightarrow \mathbb{R}^{\mu}$ such that

$$
\boldsymbol{\eta}=\boldsymbol{g}(\boldsymbol{W}),
$$

where $\boldsymbol{W}=\left(W_{1}, \ldots, W_{\nu}\right)$ is a $\nu$-dimensional zero-mean Gaussian vector such that

$$
E\left\{W_{i} W_{j}\right\}=\delta_{i j}
$$

and such that its probability density function with respect to $d \boldsymbol{w}$ is given by,

$$
p_{\boldsymbol{W}}(\boldsymbol{w})=\frac{1}{(2 \pi)^{\nu / 2}} e^{-\frac{1}{2}\|w\|_{\mathbb{R}}^{2} \nu}
$$

Moreover, noting that from Equation (??) it is deduced that,

$$
E\left\{\|\boldsymbol{\eta}\|_{\mathbb{R}^{\mu}}^{2}\right\}=\mu<+\infty,
$$

$\boldsymbol{\eta}$ admits a Chaos decomposition in terms of Gaussian variable $\boldsymbol{W}$ of the form

$$
\boldsymbol{\eta}=\sum_{\boldsymbol{\alpha},|\boldsymbol{\alpha}|=1}^{\infty} \boldsymbol{a}_{\boldsymbol{\alpha}} \phi_{\boldsymbol{\alpha}}(\boldsymbol{W}),
$$


where $\boldsymbol{\alpha}$ is a multi-index $\left(\alpha_{1}, \ldots, \alpha_{\nu}\right) \in \mathbb{N}^{\nu},|\boldsymbol{\alpha}|=\alpha_{1}+\ldots+\alpha_{\nu}, \boldsymbol{a}_{\boldsymbol{\alpha}}$ is a vector in $\mathbb{R}^{\mu}$ and $\phi_{\boldsymbol{\alpha}}(\boldsymbol{W})=\phi_{\alpha_{1}}\left(W_{1}\right) \times \ldots \times \phi_{\alpha_{\nu}}\left(W_{\nu}\right)$, in which $\phi_{\alpha_{k}}(w)$ is the normalized Hermite polynomial of order $\alpha_{k}$ such that

$$
\int_{\mathbb{R}} \phi_{\alpha_{k}}(w) \phi_{\alpha_{j}}(w) \frac{1}{\sqrt{2 \pi}} e^{-\frac{1}{2} w^{2}} d w=\delta_{\alpha_{k} \alpha_{j}} .
$$

This orthogonality condition can be generalized to the multi-indexed Hermite polynomials in the form,

$$
E\left\{\phi_{\boldsymbol{\alpha}}(\boldsymbol{W}) \phi_{\boldsymbol{\beta}}(\boldsymbol{W})\right\}=\delta_{\boldsymbol{\alpha} \boldsymbol{\beta}}=\delta_{\alpha_{1} \beta_{1}} \times \ldots \times \delta_{\alpha_{\nu} \beta_{\nu}} .
$$

The coefficients $\boldsymbol{a}_{\boldsymbol{\alpha}}$ can be clearly expressed in the form,

$$
\begin{aligned}
\boldsymbol{a}_{\boldsymbol{\alpha}} & =E\left\{\boldsymbol{\eta} \phi_{\boldsymbol{\alpha}}(\boldsymbol{W})\right\} \\
& =\int_{\mathbb{R}^{\nu}} \boldsymbol{g}(\boldsymbol{w}) \phi_{\boldsymbol{\alpha}}(\boldsymbol{w}) p_{\boldsymbol{W}}(\boldsymbol{w}) d \boldsymbol{w},
\end{aligned}
$$

which presumes knowledge of the functional form of $g($.$) . Substituting the Chaos representation$ of random variable $\boldsymbol{\eta}$ given by Eq. (??) into Eq. (??) yields the chaos representation of $\left\{\boldsymbol{U}^{\mu}(\boldsymbol{x}), \quad \boldsymbol{x} \in \mathcal{D}\right\}$,

$$
\boldsymbol{U}^{\mu}(\boldsymbol{x})=\sum_{\boldsymbol{\alpha},|\boldsymbol{\alpha}|=1}^{\infty} \boldsymbol{u}_{\boldsymbol{\alpha}}^{\mu}(\boldsymbol{x}) \phi_{\boldsymbol{\alpha}}(\boldsymbol{W})
$$

in which

$$
\boldsymbol{u}_{\boldsymbol{\alpha}}^{\mu}(\boldsymbol{x})=\sum_{j=1}^{\mu} \sqrt{\lambda_{j}}\left\{\boldsymbol{a}_{\boldsymbol{\alpha}}\right\}_{j} \boldsymbol{v}_{j}(\boldsymbol{x}),
$$

and where $\left\{\boldsymbol{a}_{\boldsymbol{\alpha}}\right\}_{j}$ denotes the $j^{\text {th }}$ component of vector $\boldsymbol{a}_{\boldsymbol{\alpha}}$. Since $E\left\{\eta_{j} \eta_{k}\right\}=\delta_{j k}$ (Eq. (??)), it can be deduced that

$$
\sum_{\boldsymbol{\alpha},|\boldsymbol{\alpha}|=1}^{\infty} \boldsymbol{a}_{\boldsymbol{\alpha}} \boldsymbol{a}_{\boldsymbol{\alpha}}^{T}=\left[I_{\mu}\right]
$$

in which $\left[I_{\mu}\right]$ is the $(\mu \times \mu)$ unit matrix.

It is noted at this point, that the parameter $\nu$ represents, essentially, the number of independent information carriers in the process $\boldsymbol{U}^{\mu}(\boldsymbol{x})$. For general processes, it is be known a-priori, and its estimated value, as described in this paper, can be used in developing reducedorder models for the phenomenon being represented.

\subsection{Finite-Dimensional Approximation and Error Estimation}

The finite chaos representation, $\boldsymbol{U}^{\mu, q}$ of order $q$, of $\boldsymbol{U}^{\mu}$ is given by

$$
\boldsymbol{U}^{\mu, q}(\boldsymbol{x})=\sum_{\boldsymbol{\alpha},|\boldsymbol{\alpha}|=1}^{q} \boldsymbol{u}_{\boldsymbol{\alpha}}^{\mu}(\boldsymbol{x}) \phi_{\boldsymbol{\alpha}}(\boldsymbol{W})
$$


From the above it can be shown that,

$$
\frac{E\left\{\left\|\boldsymbol{U}^{\mu}-\boldsymbol{U}^{\mu, q}\right\|_{H}^{2}\right\}}{E\left\{\left\|\boldsymbol{U}^{\mu}\right\|_{H}^{2}\right\}}=\frac{\sum_{j=1}^{\mu} \lambda_{j} \sum_{\boldsymbol{\alpha},|\boldsymbol{\alpha}| \geq q+1}^{\infty}\left(\left\{\boldsymbol{a}_{\boldsymbol{\alpha}}\right\}_{j}\right)^{2}}{\sum_{j=1}^{\mu} \lambda_{j}}
$$

The orthogonality of the chaos basis, $\phi_{\boldsymbol{\alpha}}$, results in

$$
\sum_{\boldsymbol{\alpha},|\boldsymbol{\alpha}| \geq q+1}^{\infty}\left\|\boldsymbol{a}_{\boldsymbol{\alpha}}\right\|_{\mathbb{R}^{\mu}}^{2}=\mu-\sum_{\boldsymbol{\alpha},|\boldsymbol{\alpha}|=1}^{q}\left\|\boldsymbol{a}_{\boldsymbol{\alpha}}\right\|_{\mathbb{R}^{\mu}}^{2}>0
$$

which, together with Equation (??), yields two types of upper bounds on the error. Since $\lambda_{1} \geq \ldots \geq \lambda_{\mu}$, the following expression can be obtained for the first bound,

$$
\frac{E\left\{\left\|\boldsymbol{U}^{\mu}-\boldsymbol{U}^{\mu, q}\right\|_{H}^{2}\right\}}{E\left\{\left\|\boldsymbol{U}^{\mu}\right\|_{H}^{2}\right\}} \leq \frac{\lambda_{1}}{\lambda_{\mu}}\left(1-\sum_{\boldsymbol{\alpha},|\boldsymbol{\alpha}|=1}^{q} \frac{1}{\mu}\left\|\boldsymbol{a}_{\boldsymbol{\alpha}}\right\|_{\mathbb{R}^{\mu}}^{2}\right) .
$$

Moreover, since

$$
\sup _{j} \sum_{\boldsymbol{\alpha},|\boldsymbol{\alpha}| \geq q+1}^{\infty}\left(\left\{\boldsymbol{a}_{\boldsymbol{\alpha}}\right\}_{j}\right)^{2} \leq \sum_{\boldsymbol{\alpha},|\boldsymbol{\alpha}| \geq q+1}^{\infty}\left\|\boldsymbol{a}_{\boldsymbol{\alpha}}\right\|_{\mathbb{R}^{\mu}}^{2}
$$

the following second upper bound is obtained,

$$
\frac{E\left\{\left\|\boldsymbol{U}^{\mu}-\boldsymbol{U}^{\mu, q}\right\|_{H}^{2}\right\}}{E\left\{\left\|\boldsymbol{U}^{\mu}\right\|_{H}^{2}\right\}} \leq \mu\left(1-\sum_{\boldsymbol{\alpha},|\boldsymbol{\alpha}|=1}^{q} \frac{1}{\mu}\left\|\boldsymbol{a}_{\boldsymbol{\alpha}}\right\|_{\mathbb{R}^{\mu}}^{2}\right) .
$$

For a given $\mu$, equations (??) and (??) permit the a-posteriori estimation of the error associated with truncating the Chaos decomposition of $\boldsymbol{U}^{\mu}$ using the known coefficients $\left\{\boldsymbol{a}_{\boldsymbol{\alpha}},|\boldsymbol{\alpha}|=1, \ldots, q\right\}$. If stochastic field $\boldsymbol{U}$ is such that the sequence of positive numbers $\lambda_{i}$ is slowly decreasing, which is the case for wideband fields for example, then Equation (??) provides a tighter bound. In this case the value of $\mu$ required to achieve adequate representation is large. If, on the other hand, stochastic field $\boldsymbol{U}$ is such that the sequence $\lambda_{i}$ is rapidly decreasing, which is the case for narrowband stochastic fields, then Equation (??) provides the tighter bound. In this case the value of $\mu$ is relatively small. It should be noted that Eq. (??) holds if Eq. (??) holds. Consequently, for $q$ fixed, if the constraint $\sum_{\boldsymbol{\alpha},|\boldsymbol{\alpha}|=1}^{q} \boldsymbol{a}_{\alpha} \boldsymbol{a}_{\alpha}^{T}=\left[I_{\mu}\right]$ is used for accelerating the convergence of the computational process for calculating coefficients $\boldsymbol{a}_{\alpha}$ then Eq. (??) does not hold. Nevertheless, the converged solution constructed with such constraint is the solution of the problem.

\subsection{Probability Distribution of the Finite-Dimensional Approximation}

The probability distribution of $\boldsymbol{\eta}$ is next expressed in terms of its Chaos coefficients $\boldsymbol{a}_{\boldsymbol{\alpha}}$. Thus the probability distribution of $\mathbb{R}^{\mu}$-valued $\boldsymbol{\eta}$ can be obtained by substituting its Chaos representation in the expression of its characteristic functional, 


$$
\begin{aligned}
\Psi_{\boldsymbol{\eta}}(\boldsymbol{b}) & =E\left\{\exp \left\{i\langle\boldsymbol{\eta}, \boldsymbol{b}\rangle_{\mathbb{R}^{\mu}}\right\}\right\} \\
& =E\left\{\exp \left\{i\left\langle\sum_{\boldsymbol{\alpha},|\boldsymbol{\alpha}|=1}^{\infty} \boldsymbol{a}_{\boldsymbol{\alpha}} \phi_{\boldsymbol{\alpha}}(\boldsymbol{W}), \boldsymbol{b}\right\rangle_{\mathbb{R}^{\mu}}\right\}\right\} \\
& =E\left\{\exp \left\{i \sum_{\boldsymbol{\alpha},|\boldsymbol{\alpha}|=1}^{\infty} \phi_{\boldsymbol{\alpha}}(\boldsymbol{W})\left\langle\boldsymbol{a}_{\boldsymbol{\alpha}}, \boldsymbol{b}\right\rangle_{\mathbb{R}^{\mu}}\right\}\right\}
\end{aligned}
$$

which can then be rewritten as,

$$
\Psi_{\boldsymbol{\eta}}(\boldsymbol{b})=\int_{\mathbb{R}^{\nu}} \exp \left\{i \sum_{\boldsymbol{\alpha},|\boldsymbol{\alpha}|=1}^{\infty} \phi_{\boldsymbol{\alpha}}(\boldsymbol{w})\left\langle\boldsymbol{a}_{\boldsymbol{\alpha}}, \boldsymbol{b}\right\rangle_{\mathbb{R}^{\mu}}\right\} p_{\boldsymbol{W}}(\boldsymbol{w}) d \boldsymbol{w} .
$$

The probability density function of $\boldsymbol{\eta}$ has thus the expression,

$$
\begin{aligned}
p_{\boldsymbol{\eta}}(\boldsymbol{y}) & =\frac{1}{(2 \pi)^{\mu}} \int_{\mathbb{R}^{\mu}} e^{-i\langle\boldsymbol{b}, \boldsymbol{y}\rangle_{\mathbb{P}} \mu} \Psi_{\boldsymbol{\eta}}(\boldsymbol{b}) d \boldsymbol{b} \\
& =\frac{1}{(2 \pi)^{\mu}} \int_{\mathbb{R}^{\mu}} \exp \left\{-i\langle\boldsymbol{b}, \boldsymbol{y}\rangle_{\mathbb{R}^{\mu}}\right\} \int_{\mathbb{R}^{\nu}} \exp \left\{i \sum_{\boldsymbol{\alpha},|\boldsymbol{\alpha}|=1}^{\infty} \phi_{\boldsymbol{\alpha}}(\boldsymbol{w})\left\langle\boldsymbol{a}_{\boldsymbol{\alpha}}, \boldsymbol{b}\right\rangle_{\mathbb{R}^{\mu}}\right\} p_{\boldsymbol{W}}(\boldsymbol{w}) d \boldsymbol{w} d \boldsymbol{b},(
\end{aligned}
$$

which can be formally rewritten as,

$$
p_{\boldsymbol{\eta}}(\boldsymbol{y})=\int_{\mathbb{R}^{\nu}} \delta_{\mathbf{0}}\left(\boldsymbol{y}-\sum_{\boldsymbol{\alpha},|\boldsymbol{\alpha}|=1}^{\infty} \phi_{\boldsymbol{\alpha}}(\boldsymbol{w}) \boldsymbol{a}_{\boldsymbol{\alpha}}\right) p_{\boldsymbol{W}}(\boldsymbol{w}) d \boldsymbol{w}
$$

in which

$$
\delta_{\mathbf{0}}(\boldsymbol{v})=\delta_{0}\left(v_{1}\right) \ldots \delta_{0}\left(v_{\mu}\right)
$$

and where $\delta_{0}($.$) is the Dirac function in \mathbb{R}$ at the origin. Truncating the Chaos decomposition of $\boldsymbol{\eta}$ at order $q$ results in the $q$-order approximation $p_{\boldsymbol{\eta}}^{q}(\boldsymbol{y})$ of $p_{\boldsymbol{\eta}}(\boldsymbol{y})$ given by

$$
\begin{aligned}
p_{\boldsymbol{\eta}}^{q}(\boldsymbol{y}) & =\frac{1}{(2 \pi)^{\mu}} \int_{\mathbb{R}^{\mu}} \exp \left\{-i\langle\boldsymbol{b}, \boldsymbol{y}\rangle_{\mathbb{R}^{\mu}}\right\} \int_{\mathbb{R}^{\nu}} \exp \left\{i \sum_{\boldsymbol{\alpha},|\boldsymbol{\alpha}|=1}^{q} \phi_{\boldsymbol{\alpha}}(\boldsymbol{w})\left\langle\boldsymbol{a}_{\boldsymbol{\alpha}}, \boldsymbol{b}\right\rangle_{\mathbb{R}^{\mu}}\right\} p_{\boldsymbol{W}}(\boldsymbol{w}) d \boldsymbol{w} d \boldsymbol{b} \\
& =\int_{\mathbb{R}^{\nu}} \delta_{\mathbf{0}}\left(\boldsymbol{y}-\sum_{\boldsymbol{\alpha},|\boldsymbol{\alpha}|=1}^{q} \phi_{\boldsymbol{\alpha}}(\boldsymbol{w}) \boldsymbol{a}_{\boldsymbol{\alpha}}\right) p_{\boldsymbol{W}}(\boldsymbol{w}) d \boldsymbol{w} .
\end{aligned}
$$

Thus from a knowledge of the Chaos coefficients $\boldsymbol{a}_{\boldsymbol{\alpha}}$ of random variable $\boldsymbol{\eta}$, its probability density function can be approximated. It is also noted that the evaluation of the integral in Equation (??) must be carried out for such a characterization to be quantified. From the second of Equations (??), it is clear that the following equation holds,

$$
p_{\boldsymbol{\eta}}^{q}(\boldsymbol{y})=E\left\{\delta_{\mathbf{0}}\left(\boldsymbol{y}-\sum_{\boldsymbol{\alpha},|\boldsymbol{\alpha}|=1}^{q} \phi_{\boldsymbol{\alpha}}(\boldsymbol{W}) \boldsymbol{a}_{\boldsymbol{\alpha}}\right)\right\}
$$


where expectation is taken relative to the $\nu$-dimensional gaussian measure. It is interesting to note that this last equation could have been written directly given that $\boldsymbol{\eta}$ has been represented by its Chaos decomposition.

\section{Statistical Representation of the Chaos Coefficients}

Least squares estimation of $\boldsymbol{a}_{\boldsymbol{\alpha}}$ yields the Hilbertian projection of $\boldsymbol{\eta}$ on $\boldsymbol{\phi}_{\boldsymbol{\alpha}}$ as indicated in Equation (??). The evaluation of this projection requires knowledge of the joint probability distribution of $\boldsymbol{W}$ and $\boldsymbol{\eta}$ which depends on the function $g($.$) which is unknown in the context$ of experimental identification. Thus least squares estimation does not provide a feasible path for estimating the Chaos coefficients from experimental evidence.

\subsection{Maximum Likelihood Method}

A probability density function of $\boldsymbol{\eta}$ in terms of parameters $\boldsymbol{a}_{\boldsymbol{\alpha}}$ has been derived in Equation (??), which is independent of function $g($.$) . The maximum likelihood method [9] can thus be$ used to estimate, from observations of $\boldsymbol{\eta}$, parameters $\boldsymbol{a}_{\boldsymbol{\alpha}}$.

Consider the probability space $(\Omega, \mathcal{T}, P)$. Let $\left(\boldsymbol{\eta}\left(\theta_{1}\right), \ldots, \boldsymbol{\eta}\left(\theta_{m}\right)\right)$ be $m$ realizations of random vector $\boldsymbol{\eta}$. The problem then is reduced to estimating $\left\{\boldsymbol{a}_{\alpha}, \quad|\alpha|=1, \ldots, q\right\}$ from observations $\left\{\boldsymbol{y}^{1}, \ldots, \boldsymbol{y}^{m}\right\}$ in which $\left(\boldsymbol{y}^{1}=\boldsymbol{\eta}\left(\theta_{1}\right), \ldots, \boldsymbol{y}^{m}=\boldsymbol{\eta}\left(\theta_{m}\right)\right)$.

Consider next the likelihood function associated with observations $\boldsymbol{y}^{1}, \ldots, \boldsymbol{y}^{m}$, given by

$$
L\left(\boldsymbol{y}^{1}, \ldots, \boldsymbol{y}^{m} ; \boldsymbol{A}\right)=p_{\boldsymbol{\eta}}\left(\boldsymbol{y}^{1}, \boldsymbol{A}\right) \times \ldots \times p_{\boldsymbol{\eta}}\left(\boldsymbol{y}^{m}, \boldsymbol{A}\right),
$$

in which $\boldsymbol{A}=\left\{\boldsymbol{a}_{\boldsymbol{\alpha}},|\boldsymbol{\alpha}|=1, \ldots, q\right\}$. The problem then can be stated as

$$
\max _{\boldsymbol{A}} L\left(\boldsymbol{y}^{1}, \ldots, \boldsymbol{y}^{m} ; \boldsymbol{A}\right) .
$$

Introducing the manifold $\mathcal{M}$ defined by Eq. (??), then the optimization problem defined by Eq. (??) can be replaced by a more efficient one:

$$
\max _{\boldsymbol{A} \in \mathcal{M}} L\left(\boldsymbol{y}^{1}, \ldots, \boldsymbol{y}^{m} ; \boldsymbol{A}\right) .
$$

However, the optimization problem defined by Eq. (??) yields a very high computational cost induced by the estimation of the joint probability density functions $p_{\boldsymbol{\eta}}\left(\boldsymbol{y}^{j}, \boldsymbol{A}\right)$, for reasonable values of the length $\mu$ of random vector $\boldsymbol{\eta}$. Consequently, it is proposed to substitute the usual likelihood function by the following one,

$$
\widetilde{L}\left(\boldsymbol{y}^{1}, \ldots, \boldsymbol{y}^{m} ; \boldsymbol{A}\right)=\left\{\prod_{j=1}^{\mu} p_{\eta_{j}}\left(y_{j}^{1}, \boldsymbol{A}\right)\right\} \times \ldots \times\left\{\prod_{j=1}^{\mu} p_{\eta_{j}}\left(y_{j}^{m}, \boldsymbol{A}\right)\right\}
$$

in which $\boldsymbol{y}^{k}=\left(y_{1}^{k}, \ldots, y_{\mu}^{k}\right)$ resulting in the following approximation to the optimization problem defined by Eq. (??)

$$
\max _{\boldsymbol{A} \in \mathcal{M}} \widetilde{L}\left(\boldsymbol{y}^{1}, \ldots, \boldsymbol{y}^{m} ; \boldsymbol{A}\right)
$$


It should be noted here that regularity of this maximization problem follows from the regularity of the representations given in equations (??) and (??). It is furthermore noted that although the surrogate likelihood function $\widetilde{L}()$ digresses to the exact likelihood $L()$ when the random variables $\eta_{i}$ are independent, equation (55) does not imply an assumption of independence between these $\eta_{i}$.

\subsection{Algorithm for ML Estimation}

Although the gradients on manifold $\mathcal{M}$ of the probability density function of $\eta_{j}$ with respect to $\boldsymbol{a}_{\boldsymbol{\alpha}}$ could be derived analytically in integral forms, they seem not be too useful given the complexity of the resulting integrals. Thus, the suggested algorithm to solve the optimization problem defined by Eq. (??) involves, in some form, the following generic steps.

1. Get $\boldsymbol{A}=\left\{\boldsymbol{a}_{\boldsymbol{\alpha}}\right\}$ with $\boldsymbol{a}_{\boldsymbol{\alpha}} \in \mathcal{M}$.

2. Estimate the probability density functions $p_{\eta_{1}}\left(y_{1}^{1}, \boldsymbol{A}\right), \ldots, p_{\eta_{\mu}}\left(y_{\mu}^{m}, \boldsymbol{A}\right)$.

3. Calculate $\widetilde{L}\left(\boldsymbol{y}^{1}, \ldots, \boldsymbol{y}^{m} ; \boldsymbol{A}\right)$.

4. Evaluate the next estimate of $\boldsymbol{a}_{\boldsymbol{\alpha}}$ in $\mathcal{M}$.

Of the above four steps, the second one is certainly the most difficult to achieve. The most efficient procedure consists in synthesizing realizations of $\boldsymbol{\eta}=\sum_{\boldsymbol{\alpha}}^{q} \boldsymbol{a}_{\boldsymbol{\alpha}} \phi_{\boldsymbol{\alpha}}(\boldsymbol{W})$, followed, for $j=1, \ldots, \mu$, by estimating $p_{\eta_{j}}\left(y_{j}^{1}, \boldsymbol{A}\right), \ldots, p_{\eta_{j}}\left(y_{j}^{m}, \boldsymbol{A}\right)$ using standard methods from mathematical statistics [?].

\section{Validation by a Numerical Simulation}

\subsection{Description of the numerical example}

We consider a boundary value problem associated with a three-dimensional elasticity problem for a bounded domain. The elastic medium has a random parameter modeled by a stochastic field (the Young modulus) whose representations will be given in section 5.2. It should be noted that the Poisson ratio should be modeled by a random field. Nevertheless, in order to simplify the present example, we have chosen to model the Poisson ratio by a constant. By using the methodology proposed in the previous sections, we will construct a representation of this stochastic field from an experimental database relative to measurements of the displacement field on the boundary of the elastic domain. For this example, the experimental database is generated by Monte Carlo numerical simulation of the stochastic boundary value problem.

\subsection{Construction of an "experimental data basis" by Monte Carlo numerical simulation of} the direct problem

5.2.1. Definition of the mechanical system. Below, we consider an elastostatic problem. The stochastic system (the structure) is constituted of an non-homogeneous isotropic linear elastic medium occupying a three-dimensional bounded domain $\mathcal{D}$ with boundary $\partial \mathcal{D}$ given in a Cartesian system $\mathrm{Ox}_{1} x_{2} x_{3}$. The geometry of domain $\mathcal{D}$ is a slender rectangular box shown in Figure 1 whose dimensions along $x_{1}, x_{2}$ and $x_{3}$ are $L_{1}=1.3 \times 10^{-1} \mathrm{~m}, L_{2}=2 \times 10^{-2} \mathrm{~m}$ and $L_{3}=2 \times 10^{-2} \mathrm{~m}$. The structure is fixed on a part $\Gamma_{0}$ of $\partial \mathcal{D}$ for which the displacement field is zero. 


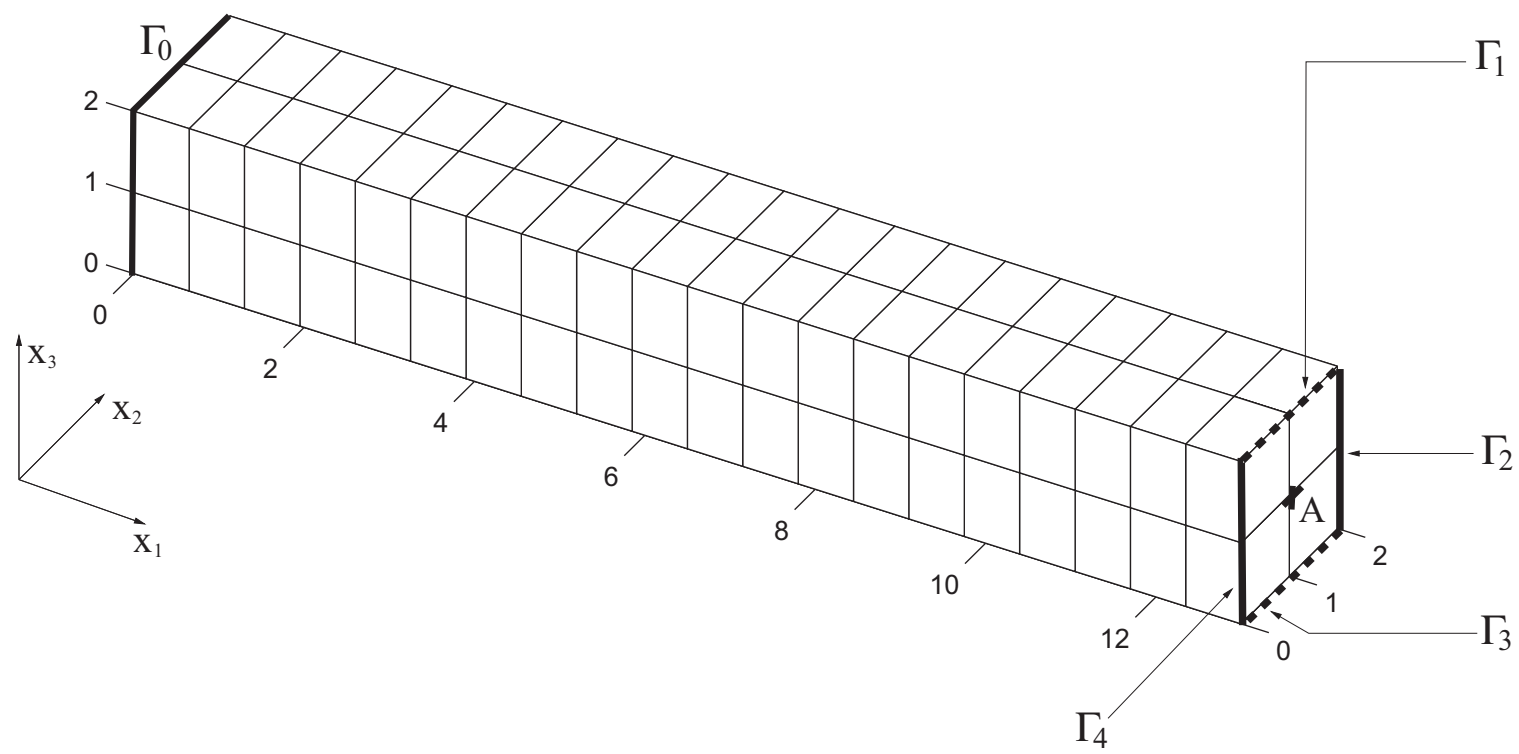

Figure 1. Definition of the mechanical system

The structure is subjected to 14 external point forces applied to the nodes in the end-section defined by $x_{1}=L_{1}$. Let $\boldsymbol{f}_{1}, \ldots, \boldsymbol{f}_{4}$ be the point forces applied to the nodes belonging to the edges $\Gamma_{1}, \ldots, \Gamma_{4}$ (Fig. 1 ) such that $\boldsymbol{f}_{1}=(0,0,1), \boldsymbol{f}_{2}=(0,1,0), \boldsymbol{f}_{3}=-\boldsymbol{f}_{1}$ and $\boldsymbol{f}_{4}=-\boldsymbol{f}_{2}$. Let $\boldsymbol{f}_{5}$ and $\boldsymbol{f}_{6}$ be the point forces applied to the nodes denoted as $A$ (Fig. 1) and such that $\boldsymbol{f}_{5}=(2,0,0)$ and $\boldsymbol{f}_{6}=(0,2,0)$.

5.2.2. Definition of the random field modeling the Young modulus. It is assumed that the only random parameter is the Young modulus which is modeled by a positive-valued second-order random field $\mathcal{E}(\boldsymbol{x})$ defined, for all $\boldsymbol{x}$ in $\mathcal{D}$, as

$$
\mathcal{E}(\boldsymbol{x})=\mathcal{E}_{0} \delta^{2} h\left(1 / \delta^{2}, V(\boldsymbol{x})\right),
$$

in which $E\{\mathcal{E}(\boldsymbol{x})\}=\mathcal{E}_{0}>0$ is the mean value independent of $\boldsymbol{x}$, where $\delta>0$ is a real parameter independent of $\boldsymbol{x}$ and allowing the dispersion level to be controlled, such that $\delta^{2}=\mathcal{E}_{0}^{-2} E\left\{\left(\mathcal{E}(\boldsymbol{x})-\mathcal{E}_{0}\right)^{2}\right\}$. The function $u \mapsto h(\alpha, u)$ from $\mathbb{R}$ into $] 0,+\infty[$ is such that $\Gamma_{\alpha}=h(\alpha, U)$ is a gamma random variable with parameter $\alpha$ if $U$ is a normalized Gaussian random variable $\left(E\{U\}=0\right.$ and $\left.E\left\{U^{2}\right\}=1\right)$. Consequently, for all $u$ in $\mathbb{R}$, we have

$$
h(\alpha, u)=F_{\Gamma_{\alpha}}^{-1}\left(F_{U}(u)\right),
$$

in which $u \mapsto F_{U}(u)=P(U \leq u)$ is the cumulative distribution function of the normalized Gaussian random variable $U$ such that

$$
F_{U}(u)=\int_{-\infty}^{u} \frac{1}{\sqrt{2 \pi}} e^{-t^{2} / 2} d t .
$$

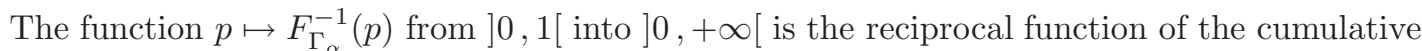
distribution function $\gamma \mapsto F_{\Gamma_{\alpha}}(\gamma)$ from $] 0,+\infty[$ into $] 0,1\left[\right.$ of the gamma random variable $\Gamma_{\alpha}$ 
with parameter $\alpha$, which is such that, for all $\gamma$ in $\mathbb{R}^{+}$,

$$
F_{\Gamma_{\alpha}}(\gamma)=\int_{0}^{\gamma} \frac{1}{\Gamma(\alpha)} t^{\alpha-1} e^{-t} d t
$$

in which $\Gamma(\alpha)=\int_{0}^{+\infty} t^{\alpha-1} e^{-t} d t$ is the gamma function. This procedure permits the simulation of positive non-gaussian processes with a marginal probability density specified as the density of a Gamma variable. In the right-hand side of Eq. (??), $\{V(\boldsymbol{x}), \boldsymbol{x} \in \mathcal{D}\}$ is a normalized Gaussian second-order random field such that $E\{V(\boldsymbol{x})\}=0$ and $E\left\{V(\boldsymbol{x})^{2}\right\}=1$, defined by

$$
V(\boldsymbol{x})=\sum_{k=1}^{3} \xi_{k} \sqrt{\gamma_{k}} \psi_{k}(\boldsymbol{x})
$$

in which $\left\{\xi_{1}, \xi_{2}, \xi_{3}\right\}$ are independent Gaussian random variables and where $\left\{\gamma_{k}\right\}_{k \geq 1}$ and $\left\{\psi_{k}\right\}_{k \geq 1}$ are defined below. In order to construct functions $\left\{\psi_{1}, \psi_{2}, \psi_{3}\right\}$, the following problem is introduced. Let $\boldsymbol{C}$ be the integral linear operator defined by the kernel $C\left(\boldsymbol{x}, \boldsymbol{x}^{\prime}\right)$ such that

$$
C\left(\boldsymbol{x}, \boldsymbol{x}^{\prime}\right)=\exp \left(-\left|x_{1}-x_{1}^{\prime}\right| / L\right),
$$

in which $L=L_{1} / 4$ and where $\boldsymbol{x}=\left(x_{1}, x_{2}, x_{3}\right)$ and $\boldsymbol{x}^{\prime}=\left(x_{1}^{\prime}, x_{2}^{\prime}, x_{3}^{\prime}\right)$ belong to $\mathcal{D}$. The eigenvalue problem related to operator $\boldsymbol{C}$ is then written as

$$
\int_{\mathcal{D}} C\left(\boldsymbol{x}, \boldsymbol{x}^{\prime}\right) \widetilde{\psi}_{k}\left(\boldsymbol{x}^{\prime}\right) d \boldsymbol{x}^{\prime}=\gamma_{k} \widetilde{\psi}_{k}(\boldsymbol{x})
$$

Since $\boldsymbol{C}$ is a Hilbert-Schmidt operator in $L^{2}(\mathcal{D}, \mathbb{R})$, the family of the eigenvalues $\left\{\gamma_{k}\right\}_{k \geq 1}$ constitutes a sequence which is assumed to be ordered by decreasing values and the family of eigenfunctions $\left\{\widetilde{\psi}_{k}\right\}_{k \geq 1}$ is a Hilbertian basis of $L^{2}(\mathcal{D}, \mathbb{R})$. Finally, the functions $\left\{\psi_{k}\right\}_{k \geq 1}$ are chosen such that

$$
\psi_{k}(\boldsymbol{x})=\frac{\widetilde{\psi}_{k}(\boldsymbol{x})}{\sqrt{\sum_{k=1}^{3} \gamma_{k} \widetilde{\psi}_{k}(\boldsymbol{x})^{2}}}
$$

Due to Eq. (??), it should be noted that Eq. (??) does not correspond to a truncated KarhunenLoeve development for which the function defined by Eq. (??) is covariance function of $V$. Figure 2 shows the graphs of 7 realizations of the stochastic field $\left\{\mathcal{E}(\boldsymbol{x}), x_{1} \in\left[0, L_{1}\right]\right\}$ obtained for $\mathcal{E}_{0}=2.1 \times 10^{10}$ N.m $m^{-2}$ and $\delta=0.2$. Since the covariance function $\left(\boldsymbol{x}, \boldsymbol{x}^{\prime}\right) \mapsto \rho\left(\boldsymbol{x}, \boldsymbol{x}^{\prime}\right)$ $\left(\boldsymbol{x}, \boldsymbol{x}^{\prime}\right) \mapsto \rho\left(\boldsymbol{x}, \boldsymbol{x}^{\prime}\right)$ of stochastic field $\left\{\mathcal{E}(\boldsymbol{x}), x_{1} \in\left[0, L_{1}\right]\right\}$ does not depend on $x_{2}, x_{2}^{\prime}, x_{3}, x_{3}^{\prime}$, this function is rewritten $\left(x_{1}, x_{1}^{\prime}\right) \mapsto \rho\left(x_{1}, x_{1}^{\prime}\right)$.

\subsection{Identification of the random field modeling the Young modulus by solving an inverse problem}

5.3.1. Construction of the experimental database. The finite element mesh of the structure is shown in Figure 1 and consists of 8-node isoparametric 3D solid finite elements. There are $N_{d}=117$ degrees of freedom. Let $\boldsymbol{\xi}=\left(\xi_{1}, \xi_{2}, \xi_{3}\right)$ be the $\mathbb{R}^{3}$-valued random variable constituted of the 3 independent random variables appearing in Equation (??) and characterizing the basic uncertainty in the problem. It is assumed that this spatial discretization is adequate for the accurate representation of the dominant eigenfunctions of the covariance kernel. Let $[A(\boldsymbol{\xi})]$ 


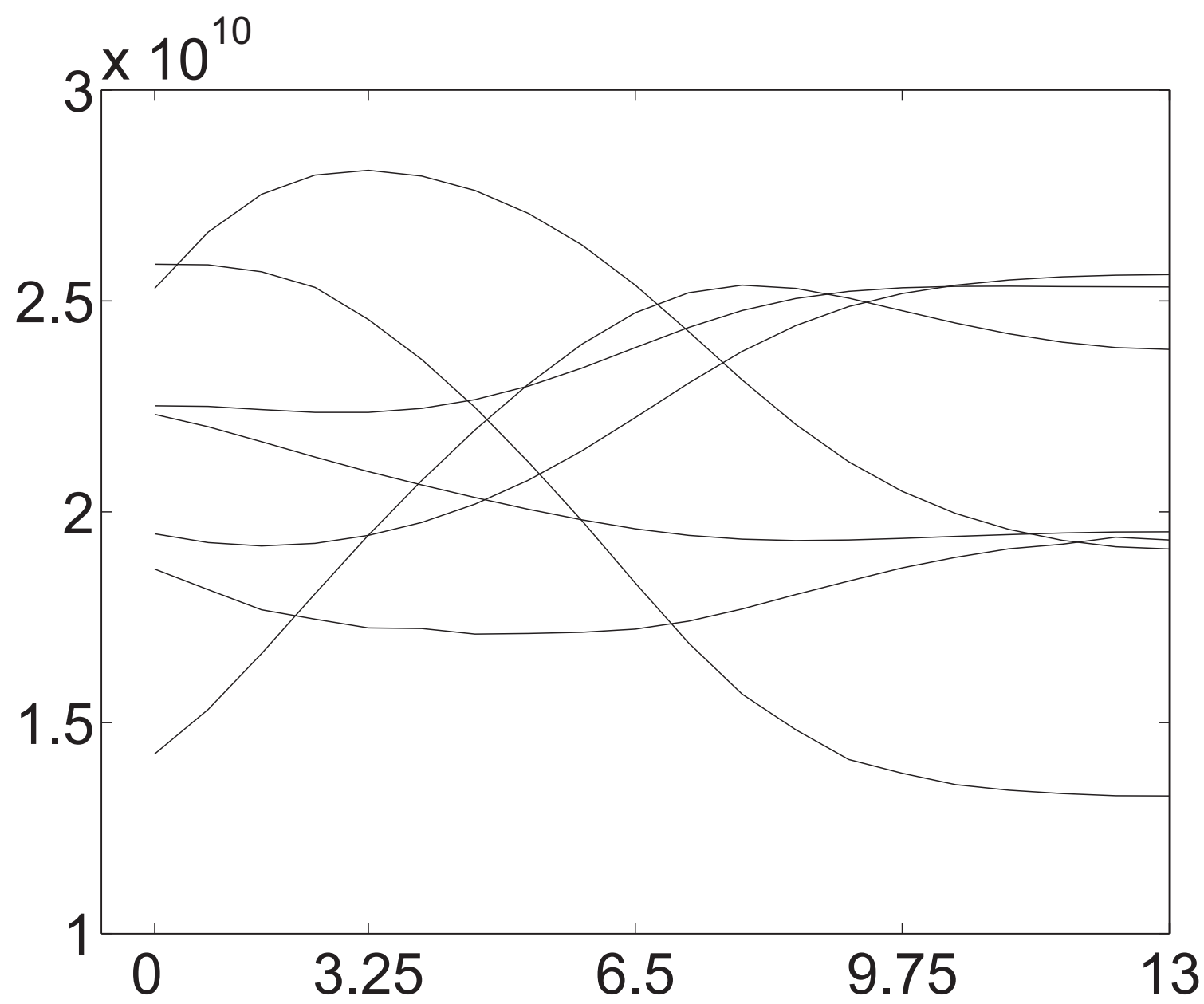

Figure 2. Graphs of 7 realizations of stochastic field $\left\{\mathcal{E}(\boldsymbol{x}), x_{1} \in\left[0, L_{1}\right]\right\}$. Horizontal axis $x_{1}$, vertical axis $\mathcal{E}(\boldsymbol{x})$.

be the random stiffness matrix with values in the set of all the positive-definite symmetric $\left(N_{d} \times N_{d}\right)$ real matrices. The $\mathbb{R}^{N_{d}}$-valued random vector of the nodal displacements is denoted by $\boldsymbol{X}=\mathbb{X}(\boldsymbol{\xi})$ and is such that

$$
[A(\boldsymbol{\xi})] \boldsymbol{X}=\boldsymbol{G}
$$

in which $G$ is the $\mathbb{R}^{N_{d}}$-vector of the external forces. The vector of the $N_{b}=81$ degrees of freedom corresponding to the nodes belonging to the boundary $\partial \mathcal{D}$ is denoted by $\boldsymbol{X}_{\partial \mathcal{D}}$. We introduce the mapping $P$ from $\mathbb{R}^{N_{d}}$ on $\mathbb{R}^{N_{b}}$ such that

$$
\boldsymbol{X}_{\partial \mathcal{D}}=P(\boldsymbol{X})
$$

The experimental data base is assumed to consist of $m=50$ random realizations of $\boldsymbol{X}_{\partial \mathcal{D}}$ which are denoted by $\boldsymbol{X}_{\partial \mathcal{D}}^{1}=\boldsymbol{X}_{\partial \mathcal{D}}\left(\theta_{1}\right), \ldots, \boldsymbol{X}_{\partial \mathcal{D}}^{m}=\boldsymbol{X}_{\partial \mathcal{D}}\left(\theta_{m}\right)$. 
5.3.2. Solving the inverse problem. The finite element approximation $\widetilde{\mathcal{E}}$ of random fields $\mathcal{E}$ indexed by $\mathcal{D}$ is such that

$$
\widetilde{\mathcal{E}}(\boldsymbol{x})=\sum_{k=1}^{N_{i}} \widetilde{\mathcal{E}}_{k} h_{k}\left(\boldsymbol{x}_{1}\right)
$$

in which $h_{1}\left(x_{1}\right), \ldots, h_{N_{i}}\left(x_{1}\right)$ are the linear interpolation functions related to the finite element mesh of domain $\mathcal{D}$, where $N_{i}=20$ is the degree of this approximation and where $\widetilde{\mathcal{E}}_{1}, \ldots \widetilde{\mathcal{E}}_{N_{i}}$ are the interpolation coefficients. We introduce the $\mathbb{R}^{N_{i}}$-valued random variable $\widetilde{\boldsymbol{\eta}}_{0}$ such that

$$
\widetilde{\boldsymbol{\eta}}_{0}=\left(\widetilde{\mathcal{E}}_{1}, \ldots, \widetilde{\mathcal{E}}_{N_{i}}\right)
$$

Let $\left[\widetilde{A}\left(\widetilde{\boldsymbol{\eta}}_{0}\right)\right]$ be the random stiffness matrix constructed using the finite element approximations $\widetilde{\mathcal{E}}(\boldsymbol{x})$ of the Young modulus. For each set of realizations $\boldsymbol{X}_{\partial \mathcal{D}}^{1}, \ldots, \boldsymbol{X}_{\partial \mathcal{D}}^{m}$ belonging to the experimental data base, the realizations

$$
\widetilde{\boldsymbol{y}}_{0}^{1}=\widetilde{\boldsymbol{\eta}}_{0}\left(\theta_{1}\right), \ldots, \widetilde{\boldsymbol{y}}_{0}^{m}=\widetilde{\boldsymbol{\eta}}_{0}\left(\theta_{m}\right),
$$

of the random variable $\widetilde{\boldsymbol{\eta}}_{0}$ are constructed by solving the following nonlinear least squares problems

$$
\begin{aligned}
\ell\left(\widetilde{\boldsymbol{y}}_{0}^{1}, \boldsymbol{X}_{\partial \mathcal{D}}^{1}\right)= & \min _{\widetilde{\boldsymbol{y}}} \ell\left(\widetilde{\boldsymbol{y}}, \boldsymbol{X}_{\partial \mathcal{D}}^{1}\right), \\
& \vdots \\
\ell\left(\widetilde{\boldsymbol{y}}_{0}^{m}, \boldsymbol{X}_{\partial \mathcal{D}}^{m}\right)= & \min _{\widetilde{\boldsymbol{y}}} \ell\left(\widetilde{\boldsymbol{y}}, \boldsymbol{X}_{\partial \mathcal{D}}^{m}\right),
\end{aligned}
$$

in which

$$
\ell(\widetilde{\boldsymbol{y}}, \boldsymbol{z})=\left\|P\left([\widetilde{A}(\widetilde{\boldsymbol{y}})]^{-1} \boldsymbol{G}\right)-\boldsymbol{z}\right\|^{2}
$$

Finally, for all $\boldsymbol{x}$ fixed in $\mathcal{D}$, the realizations $\widetilde{\mathcal{E}}^{1}(\boldsymbol{x})=\widetilde{\mathcal{E}}\left(\boldsymbol{x} ; \theta_{1}\right), \ldots, \widetilde{\mathcal{E}}^{m}(\boldsymbol{x})=\widetilde{\mathcal{E}}\left(\boldsymbol{x} ; \theta_{m}\right)$ of random variable $\widetilde{\mathcal{E}}(\boldsymbol{x})$ are constructed by using Eqs. (??) to (??). The above least squares problems are solved by using standard methods $[10,11]$.

5.3.3. Statistical reduction. The objective of the statistical reduction is to reduce the size of the random vector $\widetilde{\boldsymbol{\eta}}_{0}$ used in the representation of the random fields modeling the random Young modulus. Let $\underline{\widetilde{\boldsymbol{\eta}}} \in \mathbb{R}^{N_{i}}$ be the mean vector defined by

$$
\underline{\widetilde{\boldsymbol{\eta}}}=E\left\{\widetilde{\boldsymbol{\eta}}_{0}\right\}
$$

An estimation of vector $\underline{\widetilde{\boldsymbol{\eta}}}$ is given by

$$
\underline{\widetilde{\boldsymbol{\eta}}} \simeq \frac{1}{m} \sum_{k=1}^{m} \widetilde{\boldsymbol{y}}_{0}^{k} .
$$

Let $\widetilde{\boldsymbol{\eta}}$ be the centered $\mathbb{R}^{N_{i}}$-valued random vector defined by

$$
\widetilde{\boldsymbol{\eta}}=\widetilde{\boldsymbol{\eta}}_{0}-\underline{\tilde{\boldsymbol{\eta}}}
$$


The centered realizations $\widetilde{\boldsymbol{y}}^{1}=\widetilde{\boldsymbol{\eta}}\left(\theta_{1}\right), \ldots, \widetilde{\boldsymbol{y}}^{m}=\widetilde{\boldsymbol{\eta}}\left(\theta_{m}\right)$ of the random vector $\widetilde{\boldsymbol{\eta}}$ are then given by

$$
\begin{aligned}
\widetilde{\boldsymbol{y}}^{1} & =\widetilde{\boldsymbol{y}}_{0}^{1}-\underline{\widetilde{\boldsymbol{\eta}}}, \\
& \vdots \\
\widetilde{\boldsymbol{y}}^{m} & =\widetilde{\boldsymbol{y}}_{0}^{m}-\underline{\widetilde{\boldsymbol{\eta}}} .
\end{aligned}
$$

Let $\left[C_{\tilde{\boldsymbol{\eta}}}\right]=E\left\{\widetilde{\boldsymbol{\eta}} \widetilde{\boldsymbol{\eta}}^{T}\right\}$ be the covariance matrix of the centered random vector $\widetilde{\boldsymbol{\eta}}$ whose estimation is given by

$$
\left[C_{\widetilde{\boldsymbol{\eta}}}\right] \simeq \frac{1}{m} \sum_{k=1}^{m} \widetilde{\boldsymbol{y}}^{k} \widetilde{\boldsymbol{y}}^{k T}
$$

It should be be noted that $m$ has to be greater than $N_{i}$ in order to construct a positivedefinite matrix $\left[C_{\tilde{\boldsymbol{\eta}}}\right]$. Let $\lambda_{1} \geq \ldots \geq \lambda_{N_{i}}$ be the eigenvalues of the covariance matrix $\left[C_{\tilde{\boldsymbol{\eta}}}\right]$. The normalized eigenvectors associated with the eigenvalues $\lambda_{1}, \ldots, \lambda_{N_{i}}$ are denoted by $\boldsymbol{F}_{1}, \ldots, \boldsymbol{F}_{N_{i}}$. For all $j=1, \ldots, N_{i}$ and $\ell=1, \ldots, N_{i}$, we then have

$$
\begin{gathered}
{\left[C_{\widetilde{\boldsymbol{\eta}}}\right] \boldsymbol{F}_{j}=\lambda_{j} \boldsymbol{F}_{j},} \\
\left\langle\boldsymbol{F}_{j}, \boldsymbol{F}_{\ell}\right\rangle_{\mathbb{R}^{N_{i}}}=\delta_{j \ell} .
\end{gathered}
$$

Consequently, the random vector $\widetilde{\boldsymbol{\eta}}$ can be written as

$$
\widetilde{\boldsymbol{\eta}}=\sum_{j=1}^{N_{i}} \eta_{j} \sqrt{\lambda_{j}} \boldsymbol{F}_{j}
$$

in which $\eta_{1}, \ldots, \eta_{N_{i}}$ are $N_{i}$ centered real-valued random variables defined by $\sqrt{\lambda_{j}} \eta_{j}=$ $\left\langle\widetilde{\boldsymbol{\eta}}, \boldsymbol{F}_{j}\right\rangle_{\mathbb{R}^{N_{i}}}$ and such that for all $j$ and $k, E\left\{\eta_{j}\right\}=0$ and $E\left\{\eta_{j} \eta_{k}\right\}=\delta_{j k}$. Figure 3 displays the graph of function $k \mapsto \sum_{i=1}^{k} \lambda_{i}^{2}$.

It can be deduced that random vector $\widetilde{\boldsymbol{\eta}}$ defined by Eq. (??) can be approximated by the random vector $\widetilde{\boldsymbol{\eta}}^{\mu}$ defined by

$$
\widetilde{\boldsymbol{\eta}}^{\mu}=\sum_{j=1}^{\mu} \eta_{j} \sqrt{\lambda_{j}} \boldsymbol{F}_{j}
$$

with $\mu=5$.

We then introduce the $\mathbb{R}^{\mu}$-valued random variable $\boldsymbol{\eta}^{\mu}=\left(\eta_{1}, \ldots, \eta_{\mu}\right)$. The realizations of random vector $\boldsymbol{\eta}^{\mu}$, denoted by $\boldsymbol{y}^{1}=\boldsymbol{\eta}^{\mu}\left(\theta_{1}\right), \ldots, \boldsymbol{y}^{m}=\boldsymbol{\eta}^{\mu}\left(\theta_{m}\right)$, are calculated, for all $j=1, \ldots, \mu$, by

$$
y_{j}^{k}=\frac{1}{\sqrt{\lambda_{j}}}\left\langle\widetilde{\boldsymbol{y}}^{k}, \boldsymbol{F}_{j}\right\rangle_{\mathbb{R}^{N_{i}}} .
$$

in which, for all $k=1, \ldots, m$, we have $\boldsymbol{y}^{k}=\left(y_{1}^{k}, \ldots, y_{\mu}^{k}\right)$. 


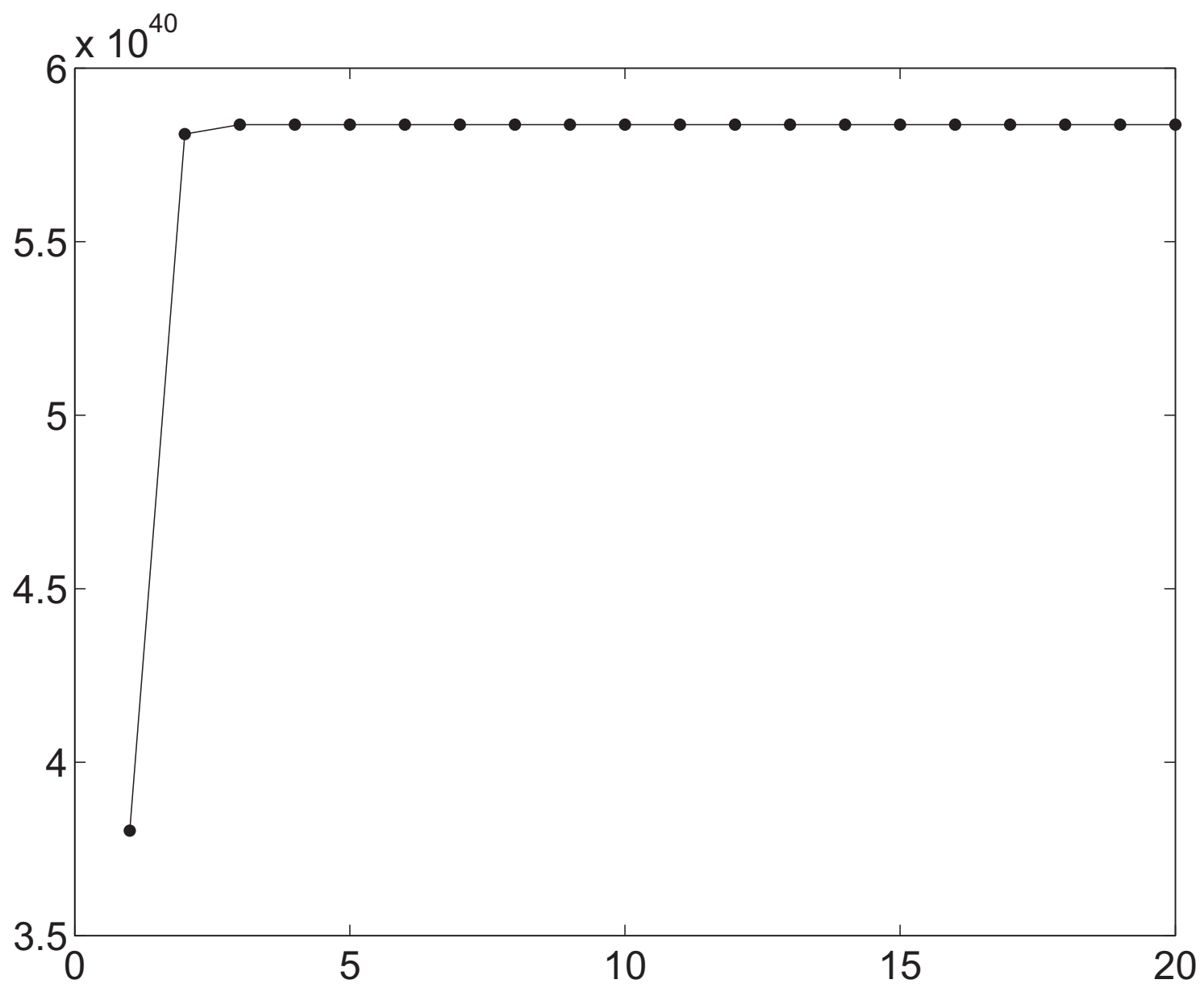

Figure 3. Convergence analysis of the statistical reduction : graph of function $k \mapsto \sum_{i=1}^{k} \lambda_{i}^{2}$. Horizontal axis $k$, vertical axis $\sum_{i=1}^{k} \lambda_{i}^{2}$.

5.3.4. Chaos decomposition. Let $\boldsymbol{W}^{\nu}=\left(W_{1}, \ldots, W_{\nu}\right)$ be the $\nu$-dimensional zero-mean Gaussian vector such that $E\left\{W_{i} W_{j}\right\}=\delta_{i j}$. The truncated Chaos representation of the $\mathbb{R}^{\mu}$ valued random variable $\boldsymbol{\eta}^{\mu}$ in terms of Gaussian variable $\boldsymbol{W}^{\nu}$ is written as

$$
\boldsymbol{\eta}^{\mu, \nu}=\sum_{\boldsymbol{\alpha},|\boldsymbol{\alpha}|=1}^{q} \boldsymbol{a}_{\boldsymbol{\alpha}} \phi_{\boldsymbol{\alpha}}\left(\boldsymbol{W}^{\nu}\right)
$$

where $\boldsymbol{\alpha}$ is a multi-index belonging to $\mathbb{N}^{\nu}$ and where $\phi_{\boldsymbol{\alpha}}\left(\boldsymbol{W}^{\nu}\right)$ is the multi-indexed Hermite polynomials (section 2.1). The coefficients $\boldsymbol{a}_{\boldsymbol{\alpha}}$ belonging to $\mathbb{R}^{\mu}$ are such that (Eq. (??))

$$
\max _{\boldsymbol{A} \in \mathcal{M}^{\mu}} \widetilde{L}\left(\boldsymbol{y}^{1}, \ldots, \boldsymbol{y}^{m} ; \boldsymbol{A}\right) \text {. }
$$


in which $\widetilde{L}$ is defined by Eq. (??), where $\boldsymbol{A}=\left\{\boldsymbol{a}_{\boldsymbol{\alpha}},|\boldsymbol{\alpha}|=1, \ldots, q\right\}$ and where $\mathcal{M}^{\mu}$ is the manifold defined by the following equation

$$
\sum_{\boldsymbol{\alpha},|\boldsymbol{\alpha}|=1}^{q} \boldsymbol{a}_{\boldsymbol{\alpha}} \boldsymbol{a}_{\boldsymbol{\alpha}}^{T}=\left[I_{\mu}\right] .
$$

It should be noted that, for all $j=1, \ldots, \mu$, Eq. (??) yields $\sum_{\boldsymbol{\alpha},|\boldsymbol{\alpha}|=1}^{q}\left\{\boldsymbol{a}_{\boldsymbol{\alpha}}\right\}_{j}^{2}=1$ which proves that, for all $\boldsymbol{\alpha}$ and for all $j$, we have

$$
-1 \leq\left\{\boldsymbol{a}_{\boldsymbol{\alpha}}\right\}_{j} \leq 1
$$

It should also be noted that since Eq. (??) has been replaced by Eq.(??), Eq. (??) does not hold (see for instance Section 3.2). Finally, random field $\{\widetilde{\mathcal{E}}(\boldsymbol{x}), \boldsymbol{x} \in \mathcal{D}\}$ defined by Eq. (??) is approximated by the random field $\widetilde{\mathcal{E}}^{\mu, \nu}(\boldsymbol{x})$ indexed by $\mathcal{D}$ and defined by

$$
\widetilde{\mathcal{E}}^{\mu, \nu}(\boldsymbol{x})=\sum_{k=1}^{N_{i}} \widetilde{\mathcal{E}}_{k}^{\mu, \nu} h_{k}\left(x_{1}\right)
$$

in which

$$
\widetilde{\mathcal{E}}_{k}^{\mu, \nu}=\underline{\boldsymbol{\eta}}_{k}+\boldsymbol{\eta}_{k}^{\mu, \nu},
$$

where $\underline{\boldsymbol{\eta}}=\left(\underline{\eta}_{1}, \ldots, \underline{\eta}_{\mu}\right)$ and $\boldsymbol{\eta}^{\mu, \nu}=\left(\eta_{1}^{\mu, \nu}, \ldots, \eta_{\mu}^{\mu, \nu}\right)$ are defined by Eqs. (??) and (??).

5.3.5. Algorithm for ML optimization. A critical step in estimating the chaos coefficients of Young modulus is to execute item 1 as specified in Section 4.2. We then have to construct a realization of random vector $\boldsymbol{A}=\left\{\boldsymbol{a}_{\boldsymbol{\alpha}}\right\}$ with $\boldsymbol{a}_{\boldsymbol{\alpha}}$ belonging to the manifold $\mathcal{M}^{\mu}$ defined by Eq. (??) and knowing that Eq. (??) holds. Let $[\boldsymbol{A}]$ be the rectangular matrix whose columns are the vectors $\boldsymbol{a}_{\boldsymbol{\alpha}}$. Consequently, the number of rows is $\mu$ and the number of columns is $\sum_{\boldsymbol{\alpha},|\boldsymbol{\alpha}|=1}^{q} 1$. Equation (??) can be rewritten as

$$
[\boldsymbol{A}][\boldsymbol{A}]^{T}=\left[I_{\mu}\right],
$$

where entries of matrix $[\boldsymbol{A}]$ are in $[-1,1]$. The proposed method for constructing matrix $[\boldsymbol{A}]$ verifying Eq. (??) is the following

1. Construction of a matrix $\left[\boldsymbol{A}_{0}\right]$ corresponding to a realization of a random matrix whose entries are independent uniform real random variables on $[-1,1]$.

2. Construction of the matrix $[\boldsymbol{A}]$ such that $[\boldsymbol{A}]=[L]^{-T}\left[\boldsymbol{A}_{0}\right]$ in which $[L]$ is the upper triangular matrix corresponding to the Cholesky decomposition $\left[\boldsymbol{B}_{0}\right]=[L]^{T}[L]$ of the matrix $\left[\boldsymbol{B}_{0}\right]=\left[\boldsymbol{A}_{0}\right]\left[\boldsymbol{A}_{0}\right]^{T}$.

It should be noted that Eq. (??) (i.e Eq. (??)) is satisfied for each iteration of the algorithm defined in Section 4.2

\subsection{Convergence Analysis}

Estimates of the mean value function $\boldsymbol{x} \mapsto \underline{\mathcal{E}}(\boldsymbol{x})=E\{\mathcal{E}(\boldsymbol{x})\}$ of stochastic field $\mathcal{E}$ are shown in Figure 4. In particular, the functions $\boldsymbol{x} \mapsto \underline{\widetilde{\mathcal{E}}}^{\mu, \nu}(\boldsymbol{x})=E\left\{\mathcal{E}^{\mu, \nu}(\boldsymbol{x})\right\}$ are shown for $\nu=1, \ldots, 8$ 

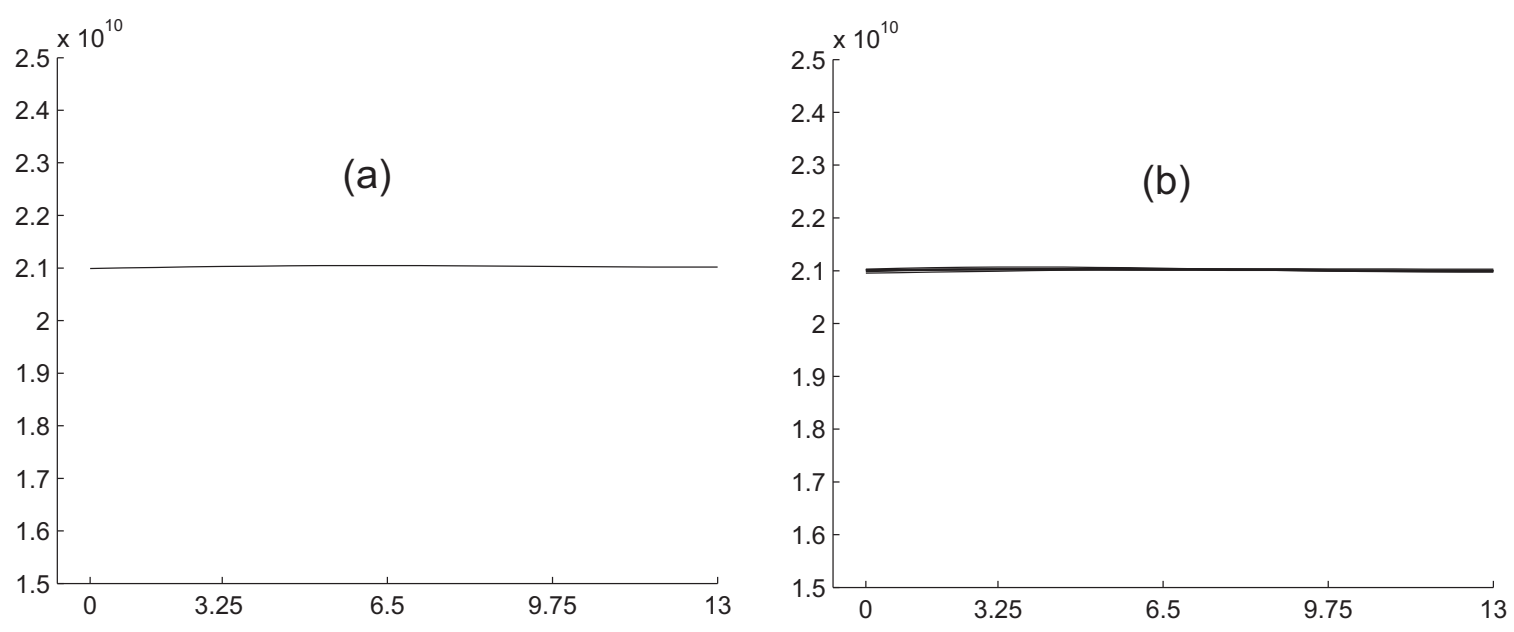

Figure 4. Convergence analysis with respect to the dimension $\nu$ of germ $\boldsymbol{W}^{\nu}$ : graph of the reference mean value function $x_{1} \mapsto \underline{\mathcal{E}}(\boldsymbol{x})$ (Fig. a) and graphs of the mean value functions $x_{1} \mapsto \widetilde{\mathcal{E}}^{\mu, \nu}(\boldsymbol{x})$ for $\nu=1, \ldots, 8$ with $\mu=5$ and $x_{2}=x_{3}=10^{-2} m$ (thick lines in Fig. b).

with $\mu=5$ and $q=6$. It should be noted that $\underline{\mathcal{E}}^{\mu, \nu}$ is independent of $q$ by construction. It can be seen that $\underline{\mathcal{E}}^{\mu, \nu}$ with $\mu=5$ is a reasonable approximation of mean value function $\underline{\mathcal{E}}$ for all $\nu$ greater or equal to 1.

In order to perform a convergence analysis of the method proposed in this paper, we introduce the normalized stochastic field $\{\varepsilon(\boldsymbol{x}), \boldsymbol{x} \in \mathcal{D}\}$ such that

$$
\varepsilon(\boldsymbol{x})=\frac{\mathcal{E}(\boldsymbol{x})}{E\{\mathcal{E}(\boldsymbol{x})\}}
$$

The finite element approximation of $\varepsilon$ is then denoted as

$$
\tilde{\mathcal{E}}^{\mu, \nu}(\boldsymbol{x})=\frac{\widetilde{\mathcal{E}}^{\mu, \nu}(\boldsymbol{x})}{E\left\{\widetilde{\mathcal{E}}^{\mu, \nu}(\boldsymbol{x})\right\}} .
$$

For all $\boldsymbol{x}$ belonging to $\mathcal{D}$, the probability density functions with respect to de of random variables $\varepsilon(\boldsymbol{x})$ and $\tilde{\varepsilon}^{\mu, \nu}(\boldsymbol{x})$ are denoted as $p_{\varepsilon(\boldsymbol{x})}(e ; \boldsymbol{x})$ and $p_{\tilde{\varepsilon}^{\mu, \nu}(\boldsymbol{x})}(e ; \boldsymbol{x})$ respectively. Some examples of probability density functions $e \mapsto p_{\tilde{\varepsilon}^{\mu, \nu}(\boldsymbol{x})}(e ; \boldsymbol{x})$ and $e \mapsto p_{\tilde{\varepsilon}^{\mu, \nu}(\boldsymbol{x})}(e ; \boldsymbol{x})$ with $\boldsymbol{x}=\left(L_{1} / 20,10^{-1}, 10^{-1}\right), \mu=5$ and for $\nu=1, \ldots, 8$ are shown in Figure 5 . These estimates of the density function were obtained from a statistical sample of size 50000 . It can be seen that a convergence is effectively reached for $\nu=3$. The graph of the reference probability density function $e \mapsto p_{\varepsilon(\boldsymbol{x})}(e ; \boldsymbol{x})$ in $\log$ scale shows that this probability density function is not gaussian.

For $\boldsymbol{x}$ in $\mathcal{D}$, the standard deviation $\sigma_{\varepsilon}(\boldsymbol{x})$ of random variable $\varepsilon(\boldsymbol{x})$ is shown in Figure 6 . In order to perform a convergence analysis of the standard deviation $\sigma_{\tilde{\varepsilon}^{\mu, \nu}}(\boldsymbol{x})$ of random variable $\tilde{\varepsilon}^{\mu, \nu}(\boldsymbol{x})$ with respect to parameter $\nu$, the graph of functions $\boldsymbol{x} \mapsto \sigma_{\tilde{\varepsilon}^{\mu, \nu}}(\boldsymbol{x})$ are also shown in Figure 6. It can be seen that the standard deviation of random variable $\varepsilon(\boldsymbol{x})$ is well approximated by $\sigma_{\tilde{\varepsilon}^{\mu, \nu}}(\boldsymbol{x})$ with $\mu=5$ and for $\nu$ greater or equal to 1 . 

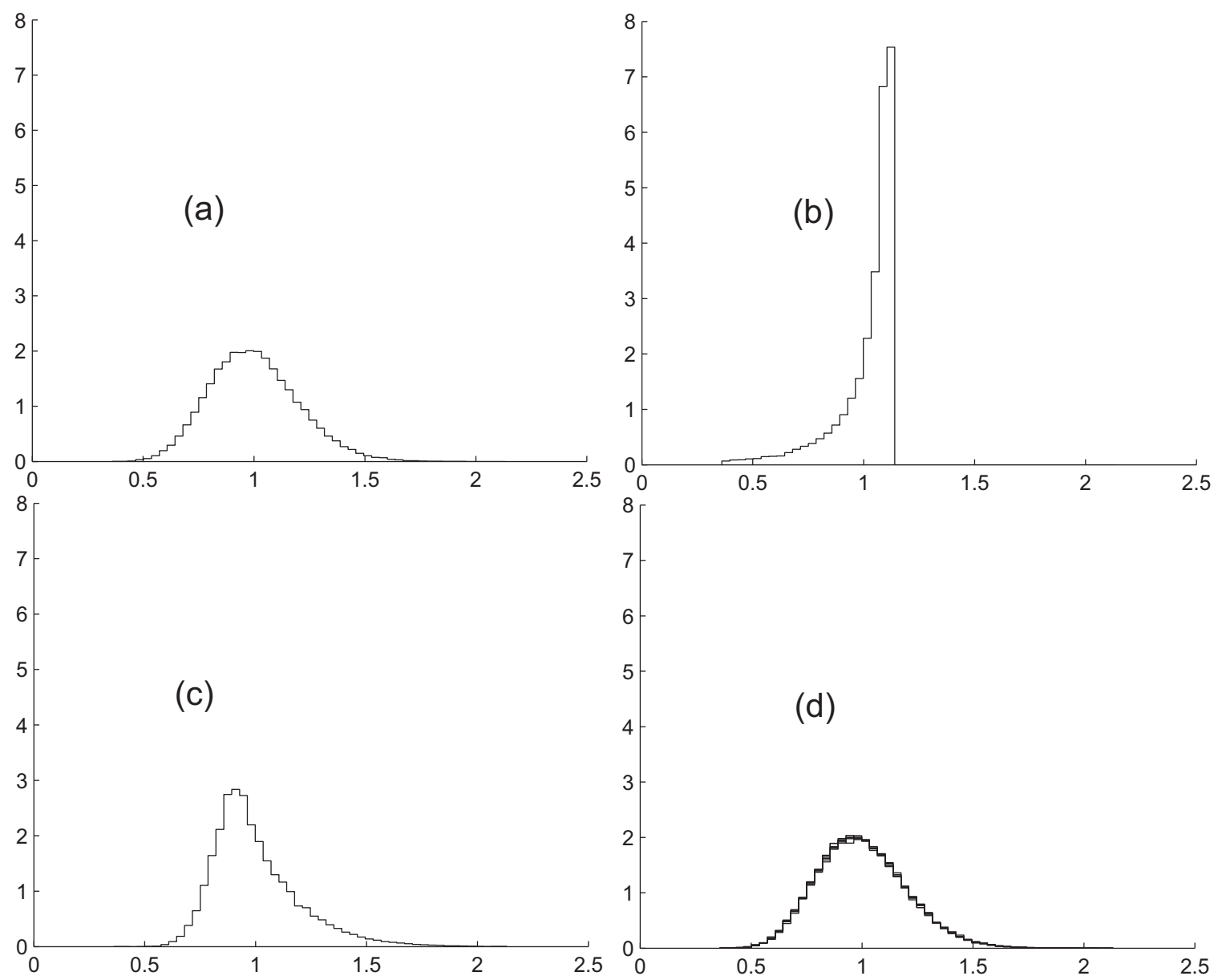

Figure 5. Examples of the probability density function of $\tilde{\varepsilon}^{\mu, \nu}$ for $\nu=1, \ldots, 8$ with $\mu=5$ : Fig. a shows the graph of the reference probability density function $e \mapsto p_{\varepsilon(\boldsymbol{x})}(e ; \boldsymbol{x})$ with $\boldsymbol{x}=\left(L_{1} / 20,10^{-1}, 10^{-1}\right)$. The graphs of $e \mapsto p_{\tilde{\varepsilon}^{\mu, \nu}(\boldsymbol{x})}(e ; \boldsymbol{x})$ with $\boldsymbol{x}=\left(L_{1} / 20,10^{-1}, 10^{-1}\right)$ are constructed for $\nu=1$ (Fig. b), $\nu=2$ (Fig. c) and $\nu=3, \ldots, 8$ (Fig. d).

For $\boldsymbol{x}$ fixed in $\mathcal{D}$, the third-order moment of random variable $\varepsilon(\boldsymbol{x})$ with is denoted as $M_{\varepsilon}^{3}(\boldsymbol{x})$. For all $\nu=1, \ldots, 8$, the third-order moment $M_{\tilde{\varepsilon}^{\mu, \nu}}^{3}(\boldsymbol{x})$ of random variable $\tilde{\varepsilon}^{\mu, \nu}(\boldsymbol{x})$ with $\mu=5$ and $q=6$ is calculated. For studying the convergence of the third-order moment of random variable $\tilde{\varepsilon}^{\mu, \nu}(\boldsymbol{x})$ with respect to $\nu$, the graphs of function $x_{1} \mapsto M_{\varepsilon}^{3}(\boldsymbol{x})$ and $x_{1} \mapsto M_{\tilde{\varepsilon} \mu, \nu}^{3}(\boldsymbol{x})$ with $\mu=5$ and for $\nu=1, \ldots, 8$ are plotted in Figure 7 . It can be seen that $M_{\widetilde{\varepsilon}^{\mu, \nu}}^{3}$ is a reasonable approximation of $M_{\varepsilon}^{3}$ constructed for $\nu$ greater or equal to 2 .

For $\boldsymbol{x}$ fixed in $\mathcal{D}$, the fourth-order moment of random variable $\varepsilon(\boldsymbol{x})$ is denoted as $M_{\varepsilon}^{4}(\boldsymbol{x})$. For all $\nu=1, \ldots, 8$, the fourth-order moment $M_{\tilde{\varepsilon}^{\mu, \nu}}^{4}(\boldsymbol{x})$ of random variable $\tilde{\varepsilon}^{\mu, \nu, q}(\boldsymbol{x})$ with $\mu=5$ and $q=6$ is calculated. For studying the convergence analysis of the fourth-order moment of random variable $\tilde{\varepsilon}^{\mu, \nu}(\boldsymbol{x})$ with respect to $\nu$, the graphs of functions $x_{1} \mapsto M_{\varepsilon}^{4}(\boldsymbol{x})$ 

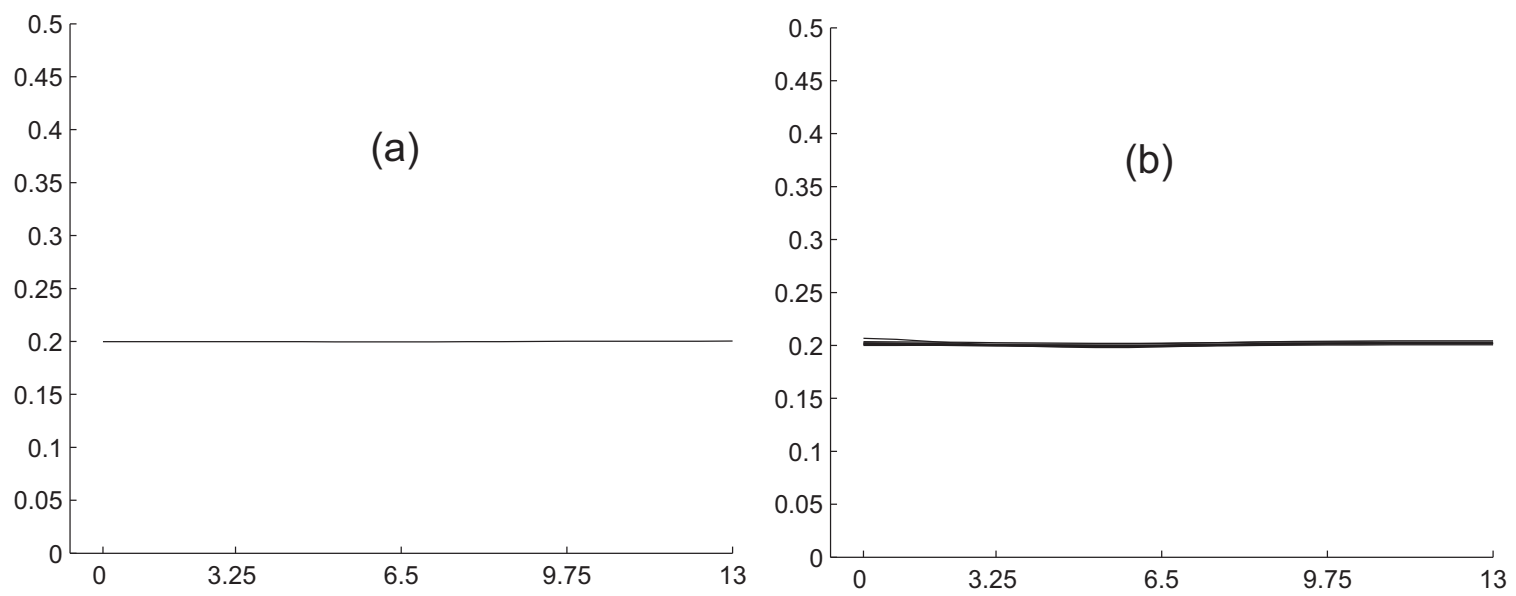

Figure 6. Convergence analysis of the standard deviation of random variable $\tilde{\varepsilon}^{\mu, \nu}(\boldsymbol{x})$ with respect to $\nu$ for all $\boldsymbol{x}$ belonging to $\mathcal{D}$ : For $x_{2}=x_{3}=10^{-2} m$, graphs of functions $x_{1} \mapsto \sigma_{\varepsilon}(\boldsymbol{x})$ (Fig. a) and $x_{1} \mapsto \sigma_{\tilde{\varepsilon}}^{\mu, \nu}(\boldsymbol{x})$ with $\mu=5$ and for $\nu=1, \ldots, 8$ (Fig. b). Horizontal axis $x_{1}$, vertical axis $\sigma_{\varepsilon}(\boldsymbol{x})$ and $\sigma_{\widetilde{\varepsilon}^{\mu, \nu}}(\boldsymbol{x})$.
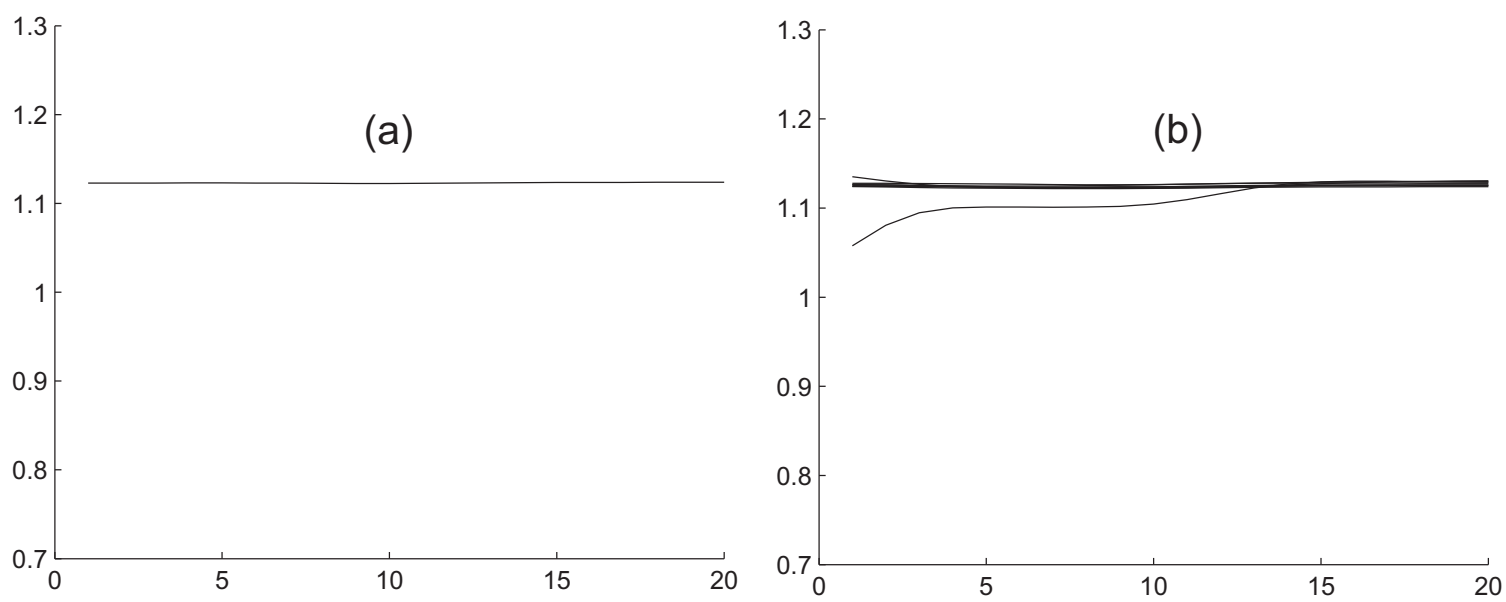

Figure 7. Convergence analysis of the third order moment of random variable $\tilde{\varepsilon}^{\mu, \nu}(\boldsymbol{x})$ with respect to $\nu$ : For $x_{2}=x_{3}=10^{-2} m$, graphs of mappings $x_{1} \mapsto M_{\varepsilon}^{3}(\boldsymbol{x})$ (Fig. a) and $x_{1} \mapsto M_{\tilde{\varepsilon} \mu, \nu}^{3}(\boldsymbol{x})$ with $\mu=5$ and for $\nu=1$ (the lower thin line in Fig. b) and $\nu=2, \ldots, 8$ (the thick lines in Fig. b). Horizontal axis $x_{1}$, vertical axis $M_{\varepsilon}^{3}(\boldsymbol{x})$ and $M_{\widetilde{\varepsilon}^{\mu}, \nu}^{3}(\boldsymbol{x})$. 

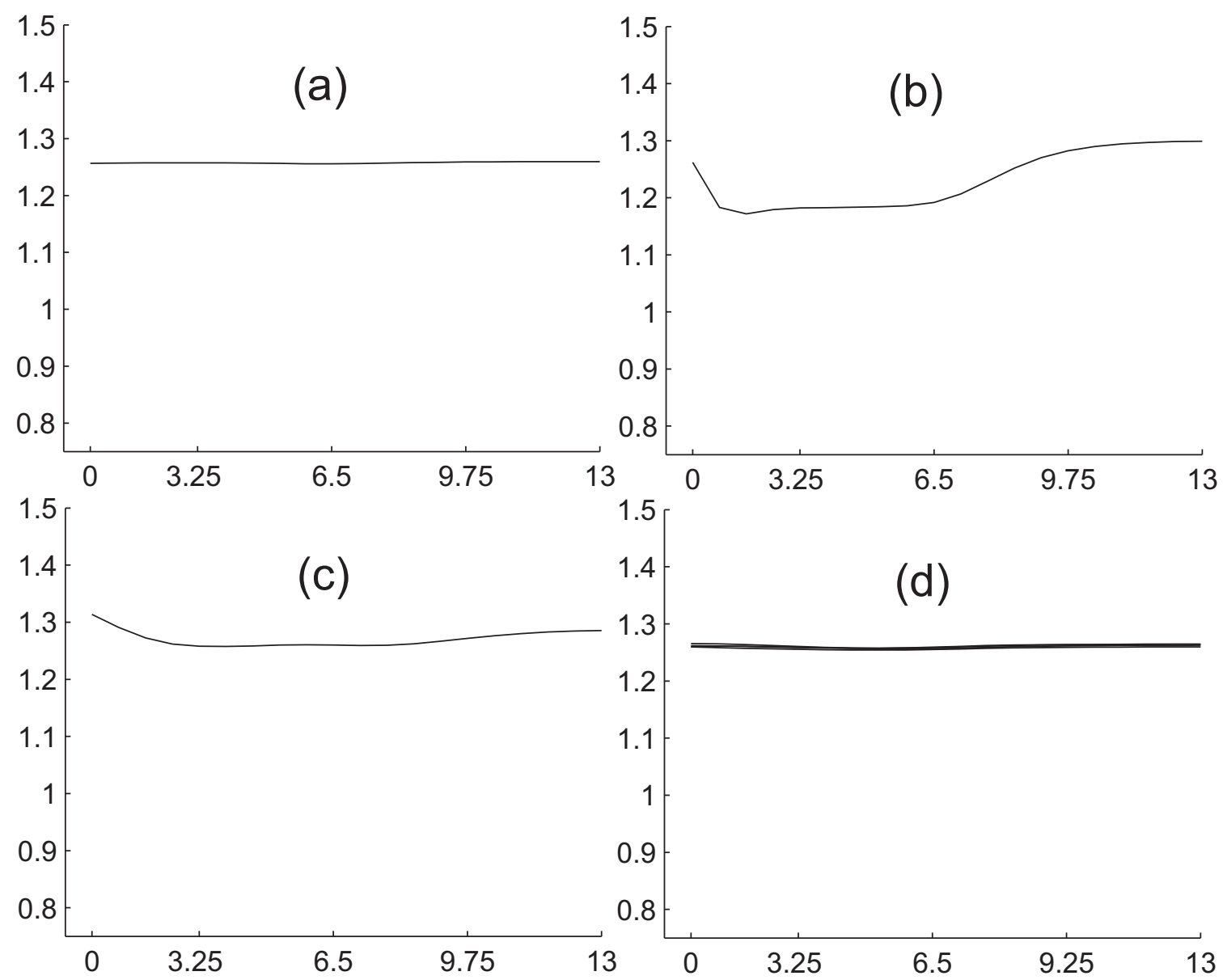

Figure 8. Convergence analysis of the fourth order moment of random variable $\tilde{\varepsilon}^{\mu, \nu}(\boldsymbol{x})$ with respect to $\nu$ : For $x_{2}=x_{3}=10^{-2} m$, graphs of functions $x_{1} \mapsto M_{\varepsilon}^{4}(\boldsymbol{x})$ (Fig. a) and $x_{1} \mapsto M_{\tilde{\varepsilon} \mu, \nu}^{4}(\boldsymbol{x})$ with $\mu=5$ and for $\nu=1$ (Fig. b), $\nu=2$ (Fig. c) and $\nu=3, \ldots, 8$ (Fig. d). Horizontal axis $x_{1}$, vertical axis $M_{\varepsilon}^{4}(\boldsymbol{x})$ and $M_{\tilde{\varepsilon} \mu, \nu}^{4}(\boldsymbol{x})$.

and $x_{1} \mapsto M_{\tilde{\varepsilon}^{\mu, \nu}}^{4}(\boldsymbol{x})$ with $\mu=5, q=6$, and for $\nu=1, \ldots, 8$ are plotted in Figure 8 . It can be seen that $M_{\tilde{\varepsilon}^{\mu, \nu}}^{4}$ is a reasonable approximation of $M_{\varepsilon}^{4}(\boldsymbol{x})$ for $\nu$ greater or equal to 3 .

Figure 9 is related to the convergence analysis of the probability functions $p_{\varepsilon^{\mu, \nu, q}(\boldsymbol{x})}(e ; \boldsymbol{x})$ with respect to the dimension $q$ of the chaos representation introduced in Eq. (84). It can be seen that $p_{\varepsilon^{\mu, \nu, q}(\boldsymbol{x})}$ is a reasonable approximation of $p_{\varepsilon(\boldsymbol{x})}$ for $q$ greater or equal to 3 .

\section{Conclusion}

A method for solving the stochastic inverse problem with chaos decomposition for experimental identification of stochastic system parameters is proposed. This method is based on the use 


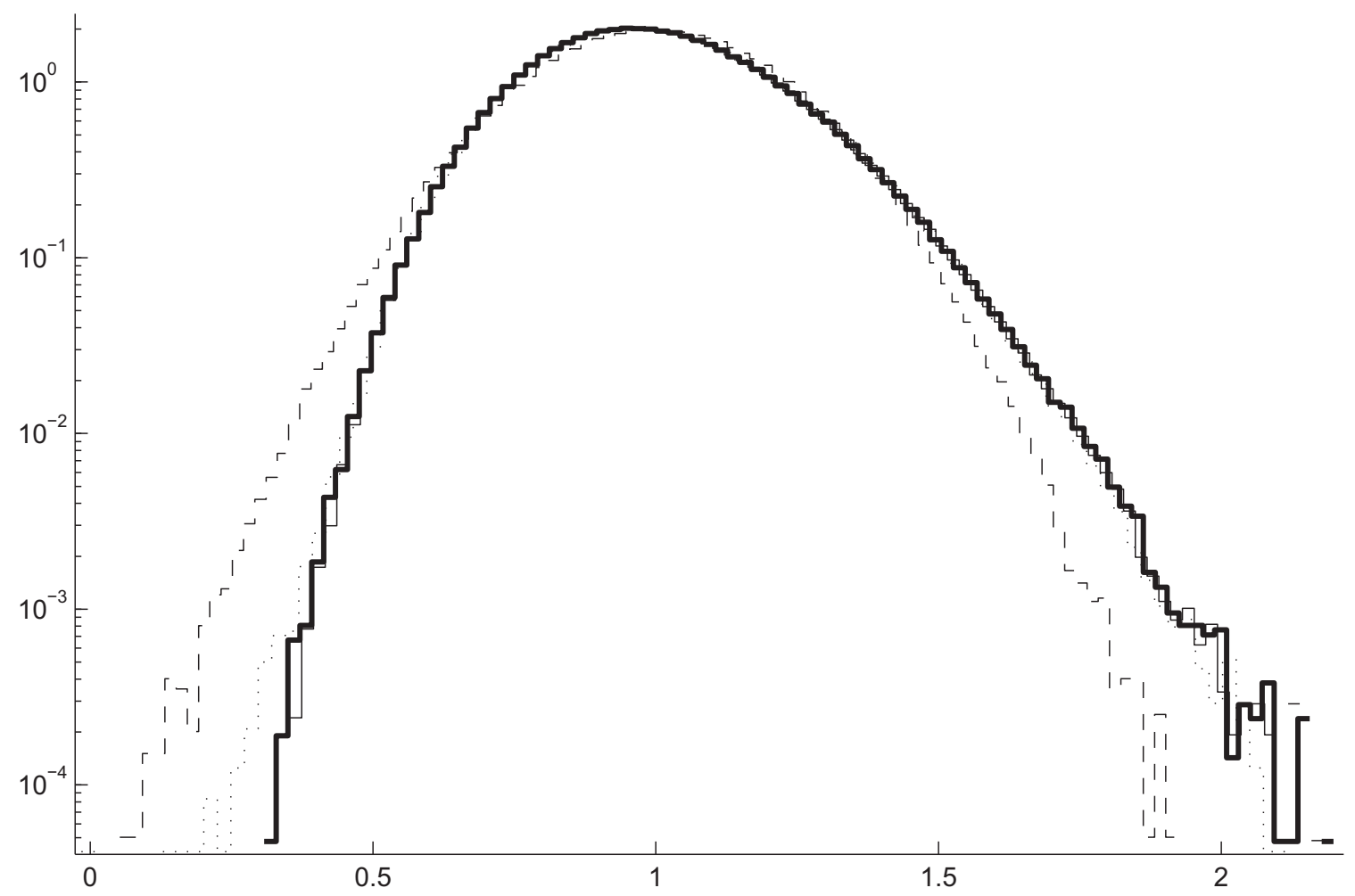

Figure 9. Convergence analysis of the probability density function of $\tilde{\varepsilon}^{\mu, \nu, q}$ with respect to the dimension $q$ of the chaos representation with $\mu=5$ and $\nu=3$ : The graphs of $e \mapsto p_{\varepsilon(\boldsymbol{x})}(e ; \boldsymbol{x})$ (thick solid line) and the graphs of $e \mapsto p_{\tilde{\varepsilon} \mu, \nu, q(\boldsymbol{x})}(e ; \boldsymbol{x})$ are constructed for $q=1$ (dashed thin line), $q=2$ (dotted thin line) and $q=3$ (solid thin line).

of chaos decomposition of the stochastic parameters to be identified and of the maximum likelihood principle. The convergence properties of this stochastic representation are studied through a numerical example. For the example considered, this method allows the probability density functions, the mean values, the standard deviation, the third and the fourth order moments to be identified. The approach presented in this paper can readily, and most beneficially, be extended to problems of structural dynamics.

\section{ACKNOWLEDGEMENT}

The financial support of the Office of Naval Research under grant number N00014-99-1-0900 is gratefully acknowledged.

\section{REFERENCES}


1. Torquato, S. (2002) Random Heterogeneous Materials, Microstructure and Macroscopic Properties, Springer-Verlag, New York.

2. Tleubergenov, M. I., (2001) "An Inverse Problem for Stochastic Differential Systems", Differential Equations, 37 (5): 751-753.

3. Angulo, J. M., and Ruiz-Medina, M. D., (1999) "Multi-Resolution Approximation to the Stochastic Inverse Problem" ,Advances in Applied Probability, 31 (4): 1039-1057.

4. Shevtsov, B. M., (1999) "Backscattering and Inverse Problem in Random Media" , Journal of Mathematical Physics, 40 (9): 4359-4373.

5. Capilla, J. E., Gomez, J. J., Sahuquillo, A., Franssen, H. J. W. M. H. (2000) "Stochastic Inverse Problems in Groundwater Modeling" ,Water Studies, 7: 295-304.

6. Wiener, N. (1938), "The Homogeneous Chaos" , American Journal of Mathematics, 60: 897-936.

7. Ghanem, R., and Spanos, P., (1991) Stochastic Finite Elements: A Spectral Approach, Springer-Verlag, New York.

8. Soize, C., and Ghanem, R. (2003) "Physical Systems with Random Uncertainties: Chaos representations with arbitrary probability measure",SIAM Journal of Scientific Computing. (in press)

9. Dennis, J. E. Jr., Nonlinear Least-Squares, State of the Art in Numerical Analysis, ed. D. Jacobs, Academic Press.

10. Marquardt D. (1963) "An Algorithm for Least-squares Estimation of Nonlinear Parameters", SIAM Journal Applied Mathematics, 11: 431-441

11. Serfling, R. J. (1980) Approximation Theorems of Mathematical Statistics, John Wiley \& Sons. 